\title{
A Survey of Digital Television Broadcast Transmission Techniques
}

\author{
Mohammed El-Hajjar and Lajos Hanzo
}

\begin{abstract}
This paper is a survey of the transmission techniques used in digital television (TV) standards worldwide. With the increase in the demand for High-Definition (HD) TV, videoon-demand and mobile TV services, there was a real need for more bandwidth-efficient, flawless and crisp video quality, which motivated the migration from analogue to digital broadcasting. In this paper we present a brief history of the development of TV and then we survey the transmission technology used in different digital terrestrial, satellite, cable and mobile TV standards in different parts of the world. First, we present the Digital Video Broadcasting standards developed in Europe for terrestrial (DVB-T/T2), for satellite (DVB-S/S2), for cable (DVB-C) and for hand-held transmission (DVB-H). We then describe the Advanced Television System Committee standards developed in the USA both for terrestrial (ATSC) and for hand-held transmission (ATSC-M/H). We continue by describing the Integrated Services Digital Broadcasting standards developed in Japan for Terrestrial (ISDB-T) and Satellite (ISDB-S) transmission and then present the International System for Digital Television (ISDTV), which was developed in Brazil by adopteding the ISDB-T physical layer architecture. Following the ISDTV, we describe the Digital Terrestrial television Multimedia Broadcast (DTMB) standard developed in China. Finally, as a design example, we highlight the physical layer implementation of the DVB-T2 standard.
\end{abstract}

Index Terms-

\section{INTRODUCTION}

$\mathbf{T}$ ELEVISION (TV) is probably the most cost-effective platform for informing, educating and entertaining people all over the globe [1-6]. The TV receiver is certainly the most popular electronic entertainment device in the world. The International Telecommunications Union (ITU) estimates the number of households with a TV set to be around 1.4 billion [7]. Radio and TV services are important for informing the public about news and information affecting their lives, as well as being a means of entertainment. Broadcasting can also serve important educational purposes by transmitting courses and other instructional material and at the time of writing, TV and radio are particularly important in countries where only few people use the Internet.

The early generations of television were mostly based on electromechanical systems, where the TV screen had a small motor with a spinning disc and a neon lamp [2,6]. In Europe, several developments occurred in the first half of the 20th

Manuscript received October 9, 2012; revised January 15, 2013. The financial support of the RC-UK under the auspices of the India-UK Advanced Technology Centre (IU-ATC), of the EU's Concerto project as well as that of the European Research Council's Advanced Fellow Grant is gratefully acknowledged.

The authors are with the School of Electronics and Computer Science, University of Southampton, SO17 1BJ, UK (e-mail: $\{$ meh,lh $\} @$ ecs.soton.ac.uk, http://www-mobile.ecs.soton.ac.uk).

Digital Object Identifier 10.1109/SURV.2013.030713.00220 century, where by the year 1950 most broadcasters were using an all-electronic system. At the same time, in the United States, several mechanical and electronic systems were developed and by 1942 the Federal Communications Commission (FCC) adopted the recommendation of the National Television System Committee (NTSC) [8].

Afterwards, various color TV systems were proposed. In Europe, the Sequential Couleur A Memoire (SECAM), French for sequential color with memory, and then the Phase Alternating Line (PAL) systems were developed and adopted by the European countries and then other countries in the world with several different variants $[1,2,6]$. In the United States, the second NTSC submitted an all-electronic color TV system to the FCC, which approved the NTSC color TV standard that was also adopted by many countries. A history of the Analogue TV enhancement projects and their development can be found in $[1,2,6]$.

Until about 1990, it was thought that implementing a digital broadcast network is impractical due to its implementation cost. However, with the advances in digital technology in both the algorithmic and hardware implementation side, broadcasters and consumer electronics manufacturers recognised the importance of switching to digital technology for improving both the bandwidth efficiency and the robustness against propagation effects using Forward Error Correction (FEC) coding. Clearly, digital technology is capable of offering numerous technical advantages over analogue broadcast as well as an enhanced user experience. The transition process is more significant than simply purchasing a set-top box to receive Digital TV (DTV). Rather, DTV supports an enhanced level of quality and flexibility unattainable by analogue broadcasting [9]. With the advances in other consumer electronics and given the requirement of providing a high-definition picture accompanied by high-quality audio, the transition to DTV was essential for the future of television.

The transition to digital technology results in an improved spectrum efficiency, where more program channels can be broadcast within the same bandwidth. This means that part of the spectrum occupied by analogue TV can be freed up for new services, such as more broadcast channels or mobile communications. This also open the way for the implementation of cognitive radio aided communications [10] in the freed frequency space, which is often referred to as TV white space. The main functions of cognitive radios can be summarised as spectrum sensing, power control and spectrum management. Several novel applications are facilitated by the employment of cognitive radio in the TV white space, such as broadband access to rural and under-served premises [11]. 
TABLE I

MAJOR DEVELOPMENTS IN THE TELEVISION HISTORY $[1,2]$.

\begin{tabular}{|c|c|}
\hline Year & Contribution \\
\hline Prior to 1900 & Several inventions were developed mostly using mechanical television designs. \\
\hline $1900-1930$ & $\begin{array}{l}\text { - The word "Television" was introduced in the World's Fair in France, where the First International Congress of } \\
\text { Electricity was held. } \\
\text { - Inventors followed two paths in the TV development: the mechanical systems and the electronics systems. } \\
\text { - Several mechanical TV systems were developed and sold in the market. } \\
\text { - During this time, there were several inventions, including the development of the cathode ray tube, an improved } \\
\text { cathode ray tube called the kinescope and the dissector tube, that formed the basis of the all-electronics TVs that } \\
\text { we use today. }\end{array}$ \\
\hline $1931-1960$ & $\begin{array}{l}\text { - The first practical electronics television system was demonstrated and TV studios were opened. } \\
\text { - Several broadcasters begin regular TV transmission. } \\
\text { - NTSC standard for black and white TV was approved by the FCC. } \\
\text { - Colour TV was invented. } \\
\text { - Cable TV was introduced. } \\
\text { - The FCC approved the NTSC color TV standard. }\end{array}$ \\
\hline $1961-1990$ & $\begin{array}{l}\text { - The first satellite to carry TV broadcasts was launched and broadcasts could be internationally relayed. } \\
\text { - PAL and SECAM were approved in Europe as the color TV standards. } \\
\text { - Most TV broadcast and TV sets in homes are in color. } \\
\text { - TV broadcast from the moon as astronaut Neil Armstrong takes mankind's first step on the moon. } \\
\text { - More regulations of the broadcasting technology and the broadcast content are introduced worldwide. } \\
\text { - Pay-TV becomes a familiar part of cable TV service. }\end{array}$ \\
\hline 1993 & The Digital Video Broadcasting for Satellite transmission (DVB-S) system was developed. \\
\hline 1994 & The Digital Video Broadcasting for Cable transmission (DVB-C) system was developed. \\
\hline 1996 & $\begin{array}{l}\text { The FCC adopted the Advanced Television System Committee (ATSC) digital television standard and mandated its use } \\
\text { for digital terrestrial television broadcasts in the United States. }\end{array}$ \\
\hline 1997 & $\begin{array}{l}\text { The Integrated Services Digital Broadcasting (ISDB) for terrestrial (ISDB-T) and satellite (ISDB-S) transmission was } \\
\text { approved in Japan. }\end{array}$ \\
\hline 2000 & The Digital Video Broadcasting for terrestrial transmission (DVB-T) system was developed. \\
\hline 2004 & The Digital Video Broadcasting for Hand-held (DVB-H) transmission was developed. \\
\hline 2005 & The second generation of Digital Video Broadcasting for Satellite transmission (DVB-S2) system was developed. \\
\hline 2006 & $\begin{array}{l}\text { - The Digital Terrestrial television Multimedia Broadcast (DTMB) was approved in China. } \\
\text { - The Brazilian International System for Digital Television (ISDTV) was published and uses the same transmission } \\
\text { technology as the ISDB-T. }\end{array}$ \\
\hline 2008 & The second generation of Digital Video Broadcasting for Terrestrial transmission (DVB-T2) system was developed. \\
\hline 2010 & The second generation of Digital Video Broadcasting for Cable transmission (DVB-C2) system was developed. \\
\hline
\end{tabular}

However, this introduces a number of technical challenges, such as spectrum sensing of both TV signals and other wireless signals as well as the ability to provide reliable service in unlicensed and dynamically changing spectral allocation scenarios [12]. Additionally, using digital technology results in an improved picture as well as sound quality and opens new opportunities for viewing TV on the move, which is referred to as mobile TV. On the other hand, as part of the ongoing digital revolution, digital TV can seamlessly interface with other communication systems, computer networks and digital media, hence enabling interactive multimedia services. Furthermore, digital TV offers a more immersive user experience with a wider choice in TV and radio channels as well as enhanced information services, including the Electronic Programming Guide and advanced teletext services [13,14].

Several digital standards were developed in different parts of the world. In Europe, broadcasters and consumer electronics companies formed a consortium for the development of digital TV. This initiative is known as Digital Video Broadcasting or DVB for short. The DVB project designed and approved several digital TV standards for terrestrial, satellite, cable and mobile TV transmission. On the other hand, following the success of the NTSC standard in the United States, the Advanced Television System Committee (ATSC) was formed for studying advanced TV solutions. This body developed the
ATSC terrestrial digital TV standard, followed by its mobile TV counterpart. In Japan, in 1984 a project was launched for designing a highly flexible standard for High Definition TV (HDTV) broadcast. The work resulted in publishing the Integrated Services Digital Broadcast (ISDB) standards for terrestrial, cable and satellite transmission. In China, another flexible digital TV standard, known as Digital Terrestrial Television Multimedia Broadcasting (DTMB), was approved in 2006. Furthermore, in 2006 the International System for Digital Television (ISDTV) was standardised as the digital TV solution in Brazil. The ISDTV physical layer is based on the ISDB-T architecture and it is now used in several countries. Table I shows a brief history of the major developments in TV broadcasting.

In this treatise we aim for providing a comprehensive survey of the transmission techniques used in the available digital TV standards in different parts of the world and then focus our attention on the most recent terrestrial TV standard, namely on the second-generation Digital Video Broadcasting for Terrestrial (DVB-T2) standard. The rest of the paper is organised as follows. In Section II, we give an overview of digital TV systems in general and then we focus our attention on the transmission technology used in the different DTV standards including the DVB standards in Section III, the ATSC standard in Section IV, the ISDB and ISDTV standards in Section V 
and finally the DTMB standard in Section VI. Then we describe the DVB-T2 physical layer design in Section VII, followed by Section VIII, where we conclude and present a vision for the future of digital broadcasting.

\section{Digital Television Systems}

As shown in Table I, the idea of transmitting pictures over the ether dates back to the 19th century and it was followed by several important developments in the 20th century that led to the invention of the all-electronics color TV. At the same time, other forms of communications were progressing and the demand for a high-quality, immersive TV experience was growing, which led to the adoption of digital broadcasting for introducing flawless high-definition video and High-Fidelity (Hi-Fi) sound as well as introducing new interactive services and designing TV systems for people on the move - or mobile TV.

Digital TV systems are composed of several standardised concepts, including video coding, audio coding, transport stream formats, middle-ware and the transmission technology. In this section we will provide a quick overview of these concepts, while the rest of the paper will focus on the transmission technology used in the different DTV standards.

\section{A. Video and Audio Coding}

At the time of writing, high definition video and audio transmission is becoming the norm in the TV industry, where the demand for high-definition video, video-on-demand and multimedia video and image services is increasing. Hence, it is essential that efficient digital compression techniques are used for encoding the video and audio signals. Considerable research efforts have been invested in the past few decades to efficiently represent video and audio signals.

Source encoding is the process of turning an analogue video/audio signal, captured by a camera for example, into a digital signal and appropriately compressing the digital signal for transmission at a reduced data rate. A source decoder carries out the reverse operations of the encoder and transforms a compressed video stream into its uncompressed format to be shown on a TV screen for example. In DTV, video and audio coding reduces the amount of bandwidth required for transmission of high-quality video and audio signals as well as for reducing the transmission power. Video/audio coding techniques are essential for reducing the transmission rate, which may however result in an erosion of the objective video/audio quality. However, the compression algorithms have to be carefully designed for ensuring that the erosion cannot be perceived by human eyes/ears [15]. This is achieved by psycho-visual and psycho-acoustic masking of the objective quality-degradation imposed by quantisation effects.

The Moving Picture Experts Group (MPEG) was formed in 1988 in order to standardise video and audio coding compression algorithms for digital storage media [4]. MPEG1 was the first MPEG standard aiming at compressing digital video and audio down to $1-1.5 \mathrm{Mbits} / \mathrm{sec}$. It was mainly targeting digital storage media such as Compact Disks (CD). MPEG-1 was then also tested for TV signal transmission and it was capable of maintaining an analogue TV signal quality.
The ubiquitous MP3 audio compression standard is based on profile 3 of the MPEG-1 standard.

MPEG-2 was then introduced in order to eliminate the weaknesses of MPEG-1 by further developing it for TV transmission. MPEG-2 standardised a higher-compression algorithm than MPEG-1, yet providing a better video quality [15, 16]. MPEG-2 is used in most of the DTV standards worldwide, including the DVB, ATSC, ISDB and DTMB solutions.

MPEG-4 was then conceived for compressing previously digitised but uncompressed video and audio signals, targeting transmission of video over the Internet or over mobile networks. MPEG-4 was also used in DTV applications, especially those using HDTV. Furthermore, MPEG-4 part 10, also known as H.264/AVC, was designed for providing MPEG2 or MPEG-4 quality video at a reduced implementation complexity. H.264/QVC is used in the most recent digital TV standards, including DVB-T2, ISDTV and DTMB.

\section{B. Data Stream Multiplexing Format}

The set of DTV signals includes video, audio and other interactive services. Their transmission is supported by control signals including the channel coding code rate and modulation scheme identities invoked for the transmission of a specific frame. Video, audio, control information and other services are transmitted in specially designed description frames right before information transmission. The most popular stream structure is the MPEG-2 audio and video Transport Stream (TS) structure. The TS is formed of a sequence of TS packets, each having a length of 188 bytes with a 4-byte header containing a synchronisation byte (sync byte) and the Packet ID (PID). MPEG also defines Program-Specific Information (PSI) that includes information about the TS payload, which is then used by the receiver for decoding the appropriate data from the stream. There is a Program Association Table (PAT) associated with a PID of ' 0 ', that includes information used by the receiver for decoding the PIDs of the Program Map Table (PMT). The PMT includes information about the PIDs for the elementary streams of each service. These streams can include audio, video and data, such as subtitles. When a receiver is decoding a specific service, first it fetches the information required for the PMT from the PAT and then the PIDs for the service from the PMT $[17,18]$.

The MPEG TS structure is used in all published DTV standards. DVB-T2 defines some other stream structures, referred to as Generic Streams (GS), in order to future-proof the standard [19].

\section{Interactive Services and Middleware}

After the Internet explosion in the 1990s, the broadcasting industry feared that their audience might shift from the traditional broadcast content to the Internet content. Hence, several innovations were introduced in the TV market, leading to the introduction of interactive TV. This meant introducing new services for giving the viewer control over the audio, video and text elements. Additionally, there is a trend for the convergence of the traditional broadcast service with Internet services, which is achieved by allowing the TV viewer to access Internet pages, video clips and Internet blogs, for 
example. Furthermore, there is a trend to provide TV content to a wide range of electronic entertainment equipment having different screen sizes. Finally, there is a trend to allow the user to interact with the TV by providing a feedback channel, where a user can interact with the TV using an enhanced remote control for example [20].

Interactive $\mathrm{TV}$ is a combination of the traditional $\mathrm{TV}$ broadcast service with the Internet. However, the convergence of these two technologies is not as simple as combining them, since the underlying technology is different in the two cases. In this case it is essential to separate the hardware and its drivers from the applications. This software layer of separation is referred to as middleware. Middleware does not have direct access to the hardware or the application, it receives input from the application via the viewer's input device such as a remote control and sends out information to the TV or to a remote device via a feedback channel [4]. Several middleware standards have been specified by different standardisation bodies, however this subject is beyond the scope of this paper and hence no more details will be provided here.

\section{Transmission Technology}

Transmission technology refers to the techniques used in the physical layer for transmitting the TV content. This includes the specific modulation and coding techniques activated as a function of the near-instantaneous wireless channel quality encountered. Digital TV was developed for terrestrial, cable, satellite and mobile channels.

The satellite transmission has a low received power, relatively high channel bandwidth and a typically Line-OfSight (LOS) transmission medium, which is only subjected to Additive White Gaussian Noise (AWGN) but no timevariant fading. On the other hand, the cable-based channel is characterised by a high Signal to Noise Ratio (SNR) and limited bandwidth, which hence suffers only from white noise but no multipath interference. The typical bandwidth provided by the coaxial cable is around $500 \mathrm{MHz}$, which is shared amongst all the TV channels sharing the cable. However, the cable's attenuation coefficient is high, hence the signals representing different $\mathrm{TV}$ channels are mapped to a $6-8 \mathrm{MHz}$ bandwidth in order to allow the cable to carry the signal for longer distances and hence to reduce the number of repeaters required [21].

The terrestrial channel is certainly the most challenging scenario among the above-mentioned systems. Terrestrial broadcasting signals experience multipath fading which results in a delay spread. Additionally, terrestrial broadcasting is expected to provide coverage for fixed receivers equipped with rooftop antennas as well as for mobile receivers relying on small built-in antennas. Therefore, the first generation of satellite and cable standards did not adopt the sophisticated and hence more robust Orthogonal Frequency Division Multiplexing (OFDM) principle [22], which was however necessitated in terrestrial broadcasting. The rest of the paper is focused on the transmission technology adopted in the different DTV standards developed in different parts of the world.

\section{Digital Video BRoAdCASTING}

During the 1980s there have been some analogue TV enhancement projects which eventually led to the formation of the DVB project. The DVB project has developed several standards for terrestrial, satellite, cable and hand-held transmission and these can be categorised as first- and second-generation standards, which will be described in the following sections.

\section{A. First Generation DVB Standards}

During the 1990s and early 2000s, the DVB project developed first-generation broadcasting standards for terrestrial, satellite, cable and hand-held transmission. These are known as the DVB-S for satellite transmission [23] developed in 1993, DVB-C for cable transmission [24] developed in 1994, DVB-T for terrestrial transmission [25] developed in 2000 and DVB-H for hand-held transmission [26] developed in 2004.

The first-generation DVB standards were designed with the following criteria in mind. First, DTV will enable the transmission of both High Definition TV (HDTV) as well as Standard Definition TV (SDTV) images via satellite, cable and terrestrial channels. Additionally, DTV will support the broadcasting of both radio programs as well as data transmission for entertainment or business purposes. Furthermore, DTV can be used to broadcast content to mobile receivers such as pocket TVs or in-vehicle TVs [27].

As shown in Table I, DVB-S was the first digital TV standard developed by the DVB project. The DVB-T and DVBC standards followed afterwards and they used a technology similar to DVB-S, with only a few modifications according to the pertinent channel characteristics in each case. Figure 1 shows the block diagram of the DVB-S, DVB-C and DVB-T transmitter. As shown in Figure 1, there are common blocks used by the three systems, including the energy dispersal ${ }^{1}$, the outer Forward Error Correction (FEC) encoder and the outer interleaver [28, 29]. The difference between the three systems is mainly due to the difference in the relevant channel characteristics between satellite, cable and terrestrial transmission as discussed in Section II-D.

All the three systems use the MPEG-2 audio and video TS structure as their data stream multiplexing format [15]. The DVB standard specifies using a multiplexer for transmitting several program channels on the same frequency. The same TS multiplexing is performed for the DVB-S, DVB-T and DVB-C standards. Figure 2(a) shows the structure of the MPEG-2 TS with 1-byte sync word and 187-byte payload. The multiplexed TS is then passed through a energy dispersal/scrambler module as shown in Figure 1, in order to generate a flat power spectral density for the stream. This is desirable in order to randomise the data stream and hence to eliminate long sequences of ' 0 's or ' 1 's. In general, it cannot be assumed that the power spectral density of a digital TV signal will be distributed evenly within the system bandwidth. More specifically, long

\footnotetext{
${ }^{1}$ The energy dispersal block is also often referred to as a scrambler or randomiser, which randomises the transmitted bit-sequence for the sake of eliminating long sequences of ' 0 ' or ' 1 ' with the aid of a Linear Feedback Shift Register (LFSR). This process increases the number of zero-crossings, which in turn supports the operation of clock-recovery. Viewing this process in the spectral domain, it results in a flattened Power Spectral Density (PSD), which justifies the 'energy-dispersal' terminology.
} 


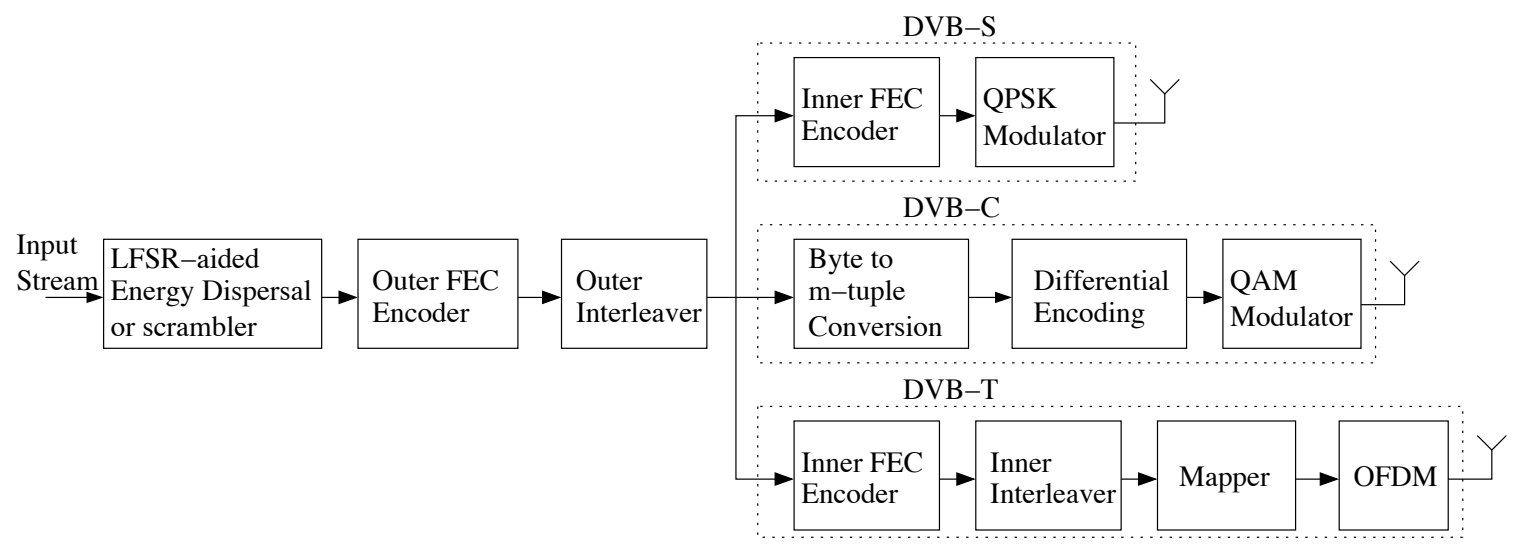

Fig. 1. Block diagram of the first generation of DVB transmitters using the parameters listed in Table II.

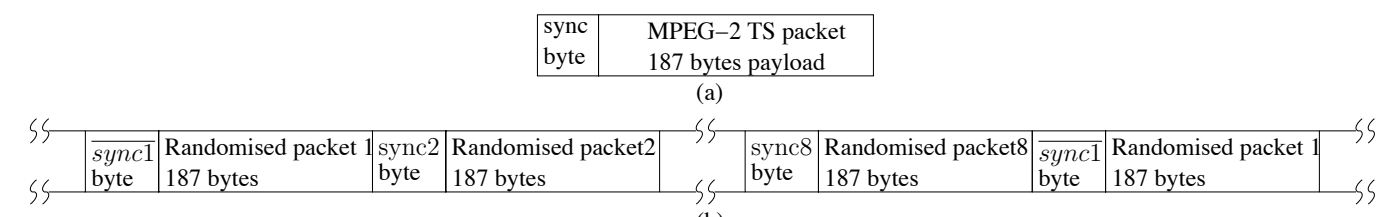

(b)

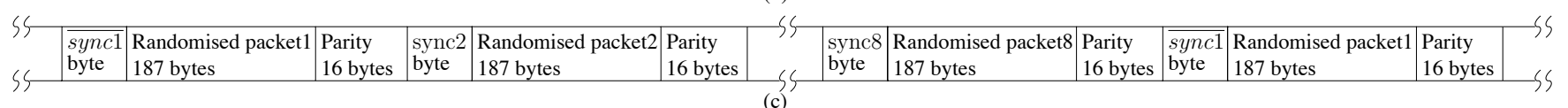

Fig. 2. Illustrations of the MPEG TS packet structure as well as the structure of the packets after energy dispersal and RS encoding in the DVB-S/C/T standards [25].

TABLE II

TRANSMITTER PARAMETERS OF THE DVB-S/C/T STANDARDS SHOWN IN FIGURE 1.

\begin{tabular}{|l|l|l|l|}
\hline Parameter & DVB-S & DVB-T & DVB-C \\
\hline LFSR polynomial & $1+X^{14}+X^{15}$ & $1+X^{14}+X^{15}$ & $1+X^{14}+X^{15}$ \\
\hline Outer FEC code & $\begin{array}{l}(204,188,8) \text { Reed-Solomon Code } \\
\text { over GF(256) }\end{array}$ & $\begin{array}{l}(204,188,8) \text { Reed-Solomon Code } \\
\text { over GF(256) }\end{array}$ & $\begin{array}{l}(204,188,8) \text { Reed-Solomon Code } \\
\text { over GF(256) }\end{array}$ \\
\hline Outer interleaver & $\begin{array}{l}\text { Convolutional interleaver with a depth } \\
\text { of } 12\end{array}$ & $\begin{array}{l}\text { Convolutional interleaver with a depth } \\
\text { of } 12\end{array}$ & $\begin{array}{l}\text { Convolutional interleaver with a depth } \\
\text { of } 12\end{array}$ \\
\hline Inner FEC code & Punctured Convolutional code & Punctured Convolutional code & - \\
\hline Inner FEC code rate & $1 / 2,2 / 3,3 / 4,5 / 6,7 / 8$ & $1 / 2,2 / 3,3 / 4,5 / 6,7 / 8$ & - \\
\hline Inner interleaver & - & $\begin{array}{l}\text { de-multiplexer followed by bit-wise } \\
\text { then symbol-wise interleaving }\end{array}$ & - \\
\hline Mapper & Gray-coded QPSK & $\begin{array}{l}\text { QPSK, 16-QAM, 64-QAM, non- } \\
\text { uniform 16-QAM, non-uniform } \\
\text { 64-QAM }\end{array}$ & $\begin{array}{l}\text { 16-QAM, 32-QAM, 64-QAM, 128- } \\
\text { QAM-QAM }\end{array}$ \\
\hline OFDM modulation & - & 2K and 4K transmission modes & - \\
\hline
\end{tabular}

sequences of ' 0 ' or ' 1 ' have rare zero-crossing and hence impose difficulties in timing recovery at the receiver [30]. Therefore, the energy dispersal block of Figure 1 is used for supporting the operation of timing recovery at the receiver and eliminating any concentration of power in a narrow spectral band, which may also lead to interference. The energy dispersal process is applied to the whole packet, except for the "sync byte", which remains untouched, in order to be used for tracking and synchronisation in the receiver. This energy dispersal uses a generator polynomial of $1+X^{14}+X^{15}$ as shown in Table II and initialises the LFSR with the bit sequence "100101010000000" [25]. The energy dispersal is applied for the whole packet, except for the "sync byte", where the LFSR is re-initialised with the initialisation sequence after every eight packets. The first packet of a sequence of eight packets has its "sync byte" inverted, in order to instruct the receiver to re-initialise the LFSR in the descrambler.

After the LFSR-aided energy dispersal, a long and powerful outer FEC encoder is used, as shown in Figure 1, which is a Reed-Solomon (RS) code [29]. This is an RS(n,k,t)=RS(204, $188,8)$ code, as shown in Table II, defined over the Galois field $\mathrm{GF}(256)$, which appends 16 parity bytes to the end of the 188 byte TS packet; hence resulting in 204-byte packets at the output of the RS encoder. An RS(n,k,t) code is capable of correcting $\mathrm{t}=(\mathrm{n}-\mathrm{k}) / 2$ symbol errors, which is $\mathrm{t}=8 \mathrm{8}$-bit symbols for the standardised RS code. Figure 2(c) shows the structure of the TS packets at the output of the RS encoder where 16 parity bytes are added to every packet resulting in 204byte packets. The channel encoder is followed by an outer convolutional interleaver of depth 12 RS-coded symbols, as shown in Figure 1 and Table II, that re-arranges the bytes in order to randomise the channel-induced errors and hence 


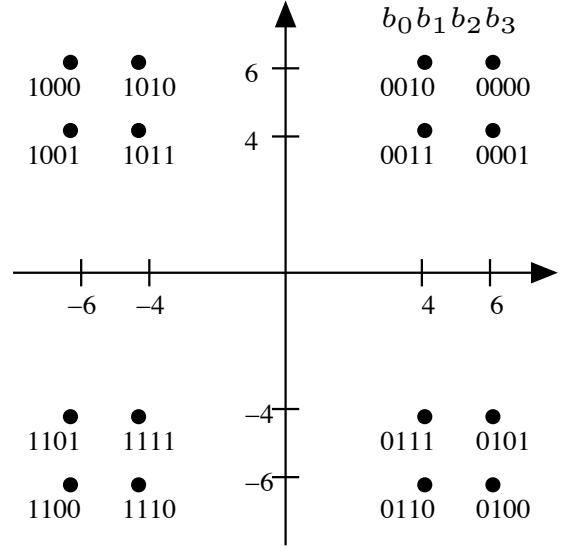

Fig. 3. Non-uniform 16-QAM constellation diagram [25].

improve the error correction capability for long bursts of errors, since the interleaver is capable of spreading the errors across the whole frame, instead of being concentrated in a single burst. The convolutional interleaver is structured in a way to keep the sync bytes as the first byte in every packet, although the structure of Figure 2 at the output of the energy dispersal block of Figure 1 is not preserved, where we had one packet having inverted sync-byte followed by 7 packets with non-inverted sync byte.

The DVB-S, DVB-C and DVB-T systems share the abovementioned three blocks of Figure 1, i.e. the energy dispersal, the outer channel encoder and the outer interleaver. Additionally, DVB-S and DVB-T use an inner FEC encoder, since the satellite and terrestrial channels typically introduce more errors than the cable-based channel. Hence, an inner FEC encoder is used in order to correct the errors imposed by both the satellite and terrestrial transmission.

1) $D V B-S$ : The inner FEC used in DVB-S is a 64-state Punctured Convolutional Code (PCC) based on a $1 / 2$-rate mother convolutional code having code rates of $1 / 2,2 / 3,3 / 4$, $5 / 6$ and $7 / 8$, as shown in Table II. The design objective of this serially concatenated structure is to ensure that the inner PCC becomes capable of beneficial soft-decisions, whilst the long outer RS code is capable of over-bridging long bursts of errors imposed by fading. However, RS codes benefit less from soft-decisions owing to the limited ability of the Chasedecoder [29]. To elaborate a little further, when the PCC is overwhelmed by channel errors, it in fact inflicts more errors than there were at the demodulator's output. The strength of the long RS code is that it may cope with this prolonged error burst, since it is not sensitive to the position of errors in its typically long codewords.

As seen in Figure 1, the DVB-S standard then maps the bits to a QPSK symbol before RF modulation and transmission. QPSK is used, since it is more robust against the non-linear distortion imposed by the power-amplifier, than 16-QAM for example, and it is indeed simple to demodulate.

2) DVB-T: As mentioned in the previous paragraph, an inner FEC encoder is used in DVB-T in order to correct the errors imposed by the terrestrial transmission. The inner FEC used in DVB-T is the same PCC as the one used in DVB-S and described in Section III-A1.
After the inner FEC, the DVB-T standard uses an inner interleaver before mapping the bits to symbols. The inner interleaver is used for further randomising the bursty errors imposed by the terrestrial channel characterised by both timeand frequency-selectivity. The inner interleaver is constituted by a combination of both bit- and symbol-interleaving and it is designed with consideration of both the affordable implementation complexity and the memory requirement of the receiver in mind. In the bit-interleaving stage of the inner interleaver seen in Figure 1, the bit stream is grouped into blocks of 126 bits, which are then interleaved in each block. Then the symbol interleaver uses a pseudo-random sequence for interleaving the blocks of 126 bits. After the interleaving, a mapper is used for mapping the bits to Gray-coded QPSK, 16QAM or 64-QAM symbols, as seen in Figure 1 and Table II. As shown in Table II, DVB-T also uses non-uniform 16QAM and non-uniform 64-QAM. An example non-uniform 16-QAM constellation is shown in Figure 3, the Euclidean distance between all the constellation points is not uniform. In a non-uniform constellation the bits have different noisesensitivity, where in the example shown in Figure 3 the bits $b_{0}$ and $b_{1}$ are the better protected bits and the bits $b_{2}$ and $b_{3}$ are the lower-protection bits, since the bits $b_{0}$ and $b_{1}$ determine the quadrant of the constellation point. This is useful when transmitting two multiplexed bit streams, where one stream carries more important information than the other. In order to decode bits $b_{0}$ and $b_{1}$, the constellation can be thought of as a QPSK constellation. Then after decoding the bits $b_{0}$ and $b_{1}$, the remaining bits will be decoded within their own quadrant. In this case, the Euclidean distance between the symbols within the four quadrants is higher than that for classic QPSK or 16QAM, which results in a better performance for the higher priority bits $b_{0}$ and $b_{1}$. However, this is achieved at the cost of a degraded $b_{3}$ and $b_{4}$, as well as overall BER.

The terrestrial channel imposes both multipath fading and delay spread, which may be mitigated by a high-complexity channel equaliser [5]. Instead, DVB-T invoked OFDM as its modulation scheme, which is capable of providing a good performance in a highly dispersive multipath channel at a reasonable implementation complexity [5,22]. OFDM achieves its robustness against dispersion by splitting the high-rate serial bit-stream into, say, 1024 reduced-rate parallel channels, which are no longer vulnerable to dispersion owing to the 1024-times longer symbols. An additional advantage of using OFDM as the modulation scheme is that it facilitates the implementation of what is referred to as a Single Frequency Network (SFN), which is used in DVB-T in order to improve the achievable spectrum efficiency [25]. In a SFN, a number of transmitters operate on the same frequency without causing interference. This means that adjacent transmitters can transmit using the same frequency, hence improving the spectral efficiency of the system, which is a great advantage, when unused frequency bands having a low path-loss at relatively low carrier frequency constitute a scarce commodity. However, it should be noted that in SFN the transmitters have to be synchronised both in time as well as frequency and they have to use the same OFDM symbol size. If these conditions are not met, then there will be interference in the system, which the OFDM receiver has a limited ability to cope with. Table III 
TABLE III

DVB-T DATA RATES IN MBit/SEC [25], EVALUATED BASED ON THE EXAMPLE SHOWN IN TABLES IV.

\begin{tabular}{|c|c|c|c|c|c|c|c|c|c|c|c|c|c|}
\hline \multirow{4}{*}{ Constellation } & \multirow{4}{*}{ Ener FEC } & \multicolumn{12}{|c|}{ Guard Interval (GI) } \\
\hline & & & & & & $\mathrm{GI}=1 / 8$ & & & $\mathrm{SI}=1 / 1$ & & & $\mathrm{SI}=1 / 32$ & \\
\hline & & \multicolumn{3}{|c|}{ Bandwidth [MHz] } & \multicolumn{3}{|c|}{ Bandwidth [MHz] } & \multicolumn{3}{|c|}{ Bandwidth [MHz] } & \multicolumn{3}{|c|}{ Bandwidth [MHz] } \\
\hline & & 6 & 7 & 8 & 6 & 7 & 8 & 6 & 7 & 8 & 6 & 7 & 8 \\
\hline \multirow{5}{*}{ QPSK } & $1 / 2$ & 3.73 & 4.35 & 4.98 & 4.14 & 4.83 & 5.53 & 4.39 & 5.12 & 5.85 & 4.52 & 5.27 & 6.03 \\
\hline & $2 / 3$ & 4.97 & 5.80 & 6.64 & 5.52 & 6.45 & 7.37 & 5.85 & 6.83 & 7.81 & 6.03 & 7.03 & 8.04 \\
\hline & $3 / 4$ & 5.59 & 6.53 & 7.46 & 6.22 & 7.25 & 8.29 & 6.58 & 7.68 & 8.78 & 6.78 & 7.91 & 9.05 \\
\hline & $5 / 6$ & 6.22 & 7.25 & 8.29 & 6.91 & 8.06 & 9.22 & 7.31 & 8.53 & 9.76 & 7.54 & 8.79 & 10.05 \\
\hline & $7 / 8$ & 6.53 & 7.62 & 8.71 & 7.25 & 8.46 & 9.68 & 7.68 & 8.96 & 10.25 & 7.91 & 9.23 & 10.56 \\
\hline \multirow{5}{*}{ 16-QAM } & $1 / 2$ & 7.46 & 8.70 & 9.95 & 8.29 & 9.67 & 11.06 & 8.78 & 10.24 & 11.71 & 9.04 & 10.55 & 1206 \\
\hline & $2 / 3$ & 9.95 & 11.61 & 13.27 & 11.05 & 12.90 & 14.75 & 11.70 & 13.66 & 15.61 & 12.06 & 14.07 & 16.09 \\
\hline & $3 / 4$ & 11.19 & 13.06 & 14.93 & 12.44 & 14.51 & 16.59 & 13.17 & 15.36 & 17.56 & 13.57 & 15.83 & 18.10 \\
\hline & $5 / 6$ & 12.44 & 14.51 & 16.59 & 13.82 & 16.12 & 18.43 & 14.63 & 17.07 & 19.52 & 15.08 & 17.59 & 20.11 \\
\hline & $7 / 8$ & 13.06 & 15.24 & 17.42 & 14.51 & 16.93 & 19.35 & 15.36 & 17.93 & 20.49 & 15.83 & 18.47 & 21.11 \\
\hline \multirow{5}{*}{ 64-QAM } & $1 / 2$ & 11.19 & 13.06 & 14.93 & 12.44 & 14.51 & 16.59 & 13.17 & 15.36 & 17.56 & 13.57 & 15.83 & 18.10 \\
\hline & $2 / 3$ & 14.92 & 17.41 & 19.91 & 16.58 & 19.35 & 22.12 & 17.56 & 20.49 & 23.42 & 18.09 & 21.11 & 24.13 \\
\hline & $3 / 4$ & 16.79 & 19.59 & 22.39 & 18.66 & 21.77 & 24.88 & 19.76 & 23.05 & 26.35 & 20.35 & 23.75 & 27.14 \\
\hline & $5 / 6$ & 18.66 & 21.77 & 24.88 & 20.73 & 24.19 & 27.65 & 21.95 & 25.61 & 27.29 & 22.62 & 26.39 & 30.16 \\
\hline & $7 / 8$ & 19.59 & 22.86 & 26.13 & 21.77 & 25.40 & 29.03 & 23.05 & 26.89 & 30.74 & 23.75 & 27.71 & 31.63 \\
\hline
\end{tabular}

TABLE IV

EXAMPLE FOR EVALUATING THE DVB-T BIT RATE USING 64-QAM, 7/8-RATE CONVOLUTIONAL CODE, 8K FFT, $1 / 32$ GUARD INTERVAL IN 8 MHZ CHANNEL. FOR MORE DETAILED ILLUSTRATION OF THE CODE RATE VALUES AND THE BANDWIDTH VALUES, PLEASE REFER TO ANNEX E IN [25].

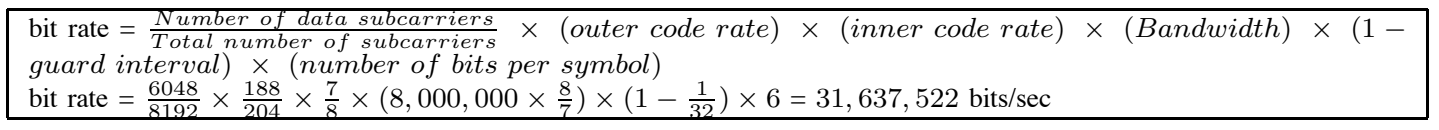

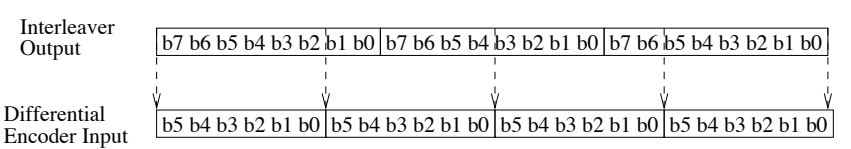

Fig. 4. Byte to m-tuple conversion of Figure 1 for the case of 64-QAM with $\mathrm{m}=6[24]$.

lists the data rates attainable by the DVB-T system, where it is shown that the DVB-T system is capable of achieving data rates in the range of 3.7 to $23.05 \mathrm{Mbit} / \mathrm{sec}$ in a $6 \mathrm{MHz}$ wide channel and in the range of 4.98 to $31.67 \mathrm{Mbit} / \mathrm{sec}$ in a $8 \mathrm{MHz}$ channel bandwidth. These data rates are valid for the DVB-T system, which were calculated after considering the appropriate FEC code rate, the constellations used and the available bandwidth, using the formula illustrated in the example of Table $\mathrm{IV}^{2}$.

3) DVB-C: As described in Section II-D, the cable channel is a transmission medium typically having a high SNR, but limited bandwidth. Hence, in DVB-C, bandwidth-efficient modulation schemes are used, including 16-QAM, 32-QAM, 64-QAM, 128-QAM and 256-QAM [5]. Observe in Figure 1 that no inner FEC is used in DVB-C, where the output of the outer FEC is mapped to a QAM symbol. However, before the bit-to-symbol mapping block of Figure 1, the output bytes of the outer interleaver ${ }^{3}$ are first converted to an m-tuple, where $\mathrm{m}$ is the number of bits per constellation symbol. For example, for $16-\mathrm{QAM}, \mathrm{m}$ is 4 , for $64-\mathrm{QAM}, \mathrm{m}$ is 6 and so on. Figure 4 shows an example of the bit to m-tuple

\footnotetext{
${ }^{2}$ The bandwidth values are normally referred to as 6,7 , and $8 \mathrm{MHz}$, but the values used in practice are included in Annex E of [25]. The practical implementation of the clock frequency is $64 / 7 \mathrm{MHz}$ for $8 \mathrm{MHz}, 48 / 7 \mathrm{MHz}$ for $6 \mathrm{MHz}$ and $8 \mathrm{MHz}$ for $7 \mathrm{MHz}$ channels [25].

${ }^{3}$ As mentioned in Section III-A, the convolutional interleaver in DVB-T/S/C operates on bytes.
}

conversion for 64 -QAM associated with $\mathrm{m}=6$. After the bit to m-tuple conversion shown in Figure 1, the two most significant bits of each symbol are differentially encoded, as shown in Figure 1, in order to obtain a constellation which is invariant to $\pi / 2$ rotations. This rotation-invariant constellation simplifies the design of the clock-synchronisation and carrier-tracking algorithms in the receiver [5]. More specifically, having a rotationally invariant constellation eliminates the false-locking problems of the carrier-recovery scheme at rotations of $n \cdot \pi / 2$, as detailed in [5].

4) DVB-H: The DVB-T standard was specified with the main objective of supporting stationary reception of terrestrial signals using roof-top antennas [18,25]. Although DVB-T was specified with sufficient flexibility to support broadcasting to mobiles, the hand-held reception was considered as desirable but not mandatory. Nonetheless, it was observed that the consumer habits have evolved by the early 2000 s and the popularity of mobile phone usage suggested the need for TV broadcast services for hand-held devices. The DVB community set about specifying a standard that is capable of delivering rich media contents to hand-held terminals, such as mobile phones. This resulted in the development of the DVB-H standard, which was designed with the following main technical requirements/challenges in mind [18, 27,31-33]:

- Hand-held terminals operate from batteries with limited capacity and hence there should be a mechanism in place for increasing the battery usage duration;

- Hand-held terminals operate with a small built-in antenna requiring the design of a robust transmission system;

- As the standard is based on a mobile broadcast system, the process of accessing services while terminals are moving from one cell to another has to be specified;

- The most grave challenge is definitely the hostile nature 


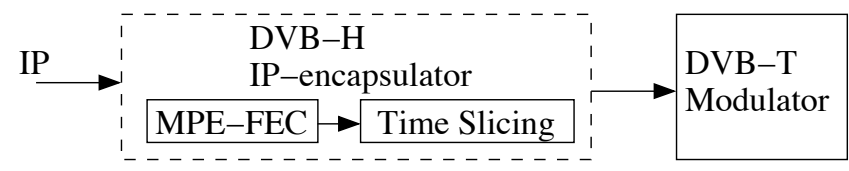

Fig. 5. Block diagram of the DVB-H transmitter, whose input is formed of Internet Protocol (IP) packets and includes Multi-Protocol Encapsulated data FEC (MPE-FEC) and time slicing in the link layer before using the same physical layer architecture as the DVB-T transmitter shown in Figure 1.

of the mobile channel, which exhibits both time- and frequency-domain fading. Additionally, the reception has to be adequate even at high speeds.

In addition to the above requirements, the DVB-H standard was to be designed as a system compatible with the already operational DVB-T system, in order to minimise the infrastructure cost for broadcasters.

As shown in Figure 5, the DVB-H standard was defined based on the existing DVB-T system, with the addition of two main components in the link layer: the Multi-Protocol Encapsulated data FEC (MPE-FEC) and time slicing [31, 32]. Time-slicing reduces the average power consumption of the terminal and enables smooth and seamless frequency handover. To elaborate a little further, time-slicing consists of sending data in bursts, where the receiver only has to 'listen' during the time slices of the requested service, otherwise it remains dormant. Additionally, time-slicing allows the receiver to monitor neighbouring cells between transmission bursts, when the receiver is off, hence supporting smooth handover both between cells as well as between services. Furthermore, MPE-FEC is also used for improving the attainable performance of the DVB-T system by improving its BER and Doppler resilience in high-speed mobile channels. Its tolerance to impulsive interference was also enhanced by the MPEFEC [31]. Explicitly, the MPE-FEC is an additional link-layer FEC scheme, which adds extra parity to those incorporated in the physical layer of the DVB-T transmitter shown in Figure 1. It is also worth mentioning that the time-slicing and MPE-FEC are implemented in the link layer and they do not affect the DVB-T physical layer architecture. Additionally, as shown in Figure 5, it is important to note that the payload of the DVB$\mathrm{H}$ signal is constituted by IP-datagrams $[31,32]$ encapsulated in the MPE data.

Table V lists the required performance of the DVB-S, DVB$\mathrm{T}$ and DVB-H systems in terms of the SNR necessitated for attaining a BER of $10^{-7}$ after the Viterbi decoder in the receiver. The performance figures listed in Table $\mathrm{V}$ are valid for a Gaussian channel and these specifications are normally issued by the standardisation bodies, such as the European Telecommunications Standards Institute (ETSI). They are also used by the receiver designers as a benchmark for their receivers' performance.

\section{B. Second Generation Standards}

Satellite transmission was the first scenario addressed by the DVB Project in 1993 and over time DVB-S has become the most popular system for the delivery of digital satellite television with more than 100 million receivers deployed worldwide. However, with the launch of HDTV services requiring more bandwidth, which was already a scarce commodity and with the increase in the transmission cost in
TABLE V

REQUIRED SIGNAL-TO-NOISE RATIO (SNR) IN DB FOR DVB-T, DVB-S AND DVB-H SYSTEMS TO ACHIEVE A BER OF $10^{-7}$ AFTER THE VITERBI DECODER AND QUASI-ERROR FREE (QEF) PERFORMANCE AFTER THE RS IN A GAUSSIAN CHANNEL [23, 25, 26].

\begin{tabular}{|l|c|c|c|c|}
\hline \multirow{2}{*}{ Constellation } & \multirow{2}{*}{ lner FEC } & \multicolumn{3}{|c|}{ Required SNR [dB] } \\
\cline { 3 - 5 } & Code Rate & DVB-T & DVB-S & DVB-H \\
\hline \hline \multirow{4}{*}{ QPSK } & $1 / 2$ & 3.5 & 4.5 & 2.6 \\
\cline { 2 - 5 } & $2 / 3$ & 5.3 & 5.0 & 4.4 \\
\cline { 2 - 5 } & $3 / 4$ & 6.3 & 5.5 & \\
\cline { 2 - 5 } & $5 / 6$ & 7.3 & 6.0 & \\
\cline { 2 - 5 } & $7 / 8$ & 7.9 & 6.4 & \\
\hline \hline \multirow{4}{*}{ 16-QAM } & $1 / 2$ & 9.3 & & 8.6 \\
\cline { 2 - 5 } & $2 / 3$ & 11.4 & & 10.7 \\
\cline { 2 - 5 } & $3 / 4$ & 12.6 & & \\
\cline { 2 - 5 } & $5 / 6$ & 13.8 & & \\
\cline { 2 - 5 } & $7 / 8$ & 14.4 & & \\
\hline \hline \multirow{5}{*}{$64-$ QAM } & $1 / 2$ & 13.8 & & 13.4 \\
\cline { 2 - 5 } & $2 / 3$ & 16.7 & & 16.3 \\
\cline { 2 - 5 } & $3 / 4$ & 18.2 & & \\
\cline { 2 - 5 } & $5 / 6$ & 19.4 & & \\
\cline { 2 - 5 } & $7 / 8$ & 20.2 & & \\
\hline
\end{tabular}

addition to increasing the number of broadcast channels, the DVB community issued a second generation satellite standard DVB-S2 [34] in order to increase the capacity of satellite broadcasting services [35]. The goal was to increase the data throughput in a given bandwidth or, alternatively, to use less satellite capacity and hence to save costs. Additionally, it was required to design a more robust system with increased availability through improved link margin and increased coverage area with smaller receiver dishes. DVB-S2 is capable of providing up to $30 \%$ of capacity gain over DVB-S.

In DVB-S, QPSK was the highest-order constellation used, which was a limitation for professional applications equipped with larger antennas that were potentially capable of supporting higher-order constellations and higher data rates. Hence, in support of the higher order modulation schemes conceived for satellite news gathering, the DVB Digital Satellite News Gathering (DVB-DSNG) standard was created [35]. However, with the addition of 8PSK and QAM schemes to deliver a high-throughput, the need for more robust error correction methods capable of robust operation at reduced $E_{b} / N_{0}$ thresholds became apparent and an improved successor to the ReedSolomon/Viterbi FEC scheme was contemplated [35].

All this led to the development of a second-generation satellite broadcast system known as DVB-S2. The DVBS2 standard was specified according to the following priorities: best transmission performance, total flexibility and reasonable receiver complexity. In order to strike an attractive performance-complexity trade-off, which also achieved a $30 \%$ capacity gain by DVB-S2 over DVB-S, DVB-S2 was designed by exploiting all the advances in both channel coding and modulation as well as in hardware technology [36]. The 
TABLE VI

PARAMETER COMPARISON OF DVB-T AND DVB-T2 AND USING THE EXAMPLE IN TABLE IV FOR ILLUSTRATING THE EVALUATION OF THE MAXIMUM BIT-RATE.

\begin{tabular}{|l|l|l|}
\hline & DVB-T & DVB-T2 \\
\hline FEC & Reed-Solomon and Convolutional Codes & BCH and LDPC codes \\
\hline Inner FEC rates & $1 / 2,2 / 3,3 / 4,5 / 6,7 / 8$ & $1 / 2,3 / 5,2 / 3,3 / 4,4 / 5,5 / 6$, \\
\hline Modulation & QPSK, 16-QAM, 64-QAM & QPSK, 16-QAM, 64-QAM, 256-QAM \\
\hline FFT size & $2 \mathrm{~K}, 8 \mathrm{~K}$ & $\begin{array}{l}1 \mathrm{~K}, 2 \mathrm{~K}, 4 \mathrm{~K}, 8 \mathrm{~K}, 8 \mathrm{~K} \text { ext., 16K, 16K ext., 32K, 32K } \\
\text { ext. }\end{array}$ \\
\hline Number of data subcarriers & 1512 for $2 \mathrm{~K}, 6048$ for $8 \mathrm{~K}$ & $764-27404$ for $1 \mathrm{~K}$ to $32 \mathrm{~K}$ with all pilot patterns \\
\hline Guard interval & $1 / 4,1 / 8,1 / 16,1 / 32$ & $1 / 4,19 / 256,1 / 8,19 / 128,1 / 16,1 / 32,1 / 128$ \\
\hline Pilots overhead & $10.5 \%$ & $1.5 \%-8.5 \%$ \\
\hline Bandwidth $\boldsymbol{B}[\mathrm{MHz}]$ & $5,6,7,8$ & $1.7,5,6,7,8,10$ \\
\hline Max, bit rate $\boldsymbol{R}[\mathrm{Mbit} / \mathrm{sec}]$ & $31.63 \mathrm{Mbit} / \mathrm{sec}$ & $50.34 \mathrm{Mbit} / \mathrm{sec}$ \\
\hline $\begin{array}{l}\text { Max. bandwidth efficiency } \mathbf{R} \\
8 \mathrm{MHz} \text { channel }\end{array}$ & $3.95 \mathrm{bits} / \mathrm{sec} / \mathrm{Hz}$ & $6.29 \mathrm{bits} / \mathrm{sec} / \mathrm{Hz}$ \\
\hline
\end{tabular}

TABLE VII

PARAMETER COMPARISON OF DVB-S, DVB-DSNG AND DVB-S2.

\begin{tabular}{|c|c|c|c|}
\hline & DVB-S & DVB-DSNG & DVB-S2 \\
\hline FEC & RS and Convolutional Codes & RS and Convolutional Codes & $\mathrm{BCH}$ and LDPC codes \\
\hline Inner FEC Rates & $1 / 2,2 / 3,3 / 4,5 / 6,7 / 8$ & $1 / 2,2 / 3,3 / 4,5 / 6,7 / 8$ & $\begin{array}{l}1 / 4,1 / 3,2 / 5,1 / 2,3 / 5,2 / 3, \\
3 / 4,4 / 5,5 / 6,8 / 9,9 / 10\end{array}$ \\
\hline Total FEC Rates & $0.46,0.61,0.69,0.76,0.80$ & $0.46,0.61,0.69,0.76,0.80$ & $\begin{array}{l}0.23,0.30,0.36,0.46,0.55,0.61 \\
0.69,0.73,0.76,0.81,0.83\end{array}$ \\
\hline Modulation & BPSK, QPSK & BPSK, QPSK, 16-QAM & $\begin{array}{l}\text { QPSK, } \\
\text { APSK }\end{array}$ \\
\hline Nyquist Roll-off factor & 0.35 & $0.25,0.35$ & $0.2,0.25,0.35$ \\
\hline Pilots overhead & - & - & $2 \%$ \\
\hline $\begin{array}{l}\text { Maximum bandwidth Efficiency } \\
\text { using a roll-off factor of ' } 0 \text { ' }\end{array}$ & $1.61 \mathrm{bits} / \mathrm{sec} / \mathrm{Hz}$ & $3.22 \mathrm{bits} / \mathrm{sec} / \mathrm{Hz}$ & $4.44 \mathrm{bits} / \mathrm{sec} / \mathrm{Hz}$ \\
\hline $\begin{array}{l}\text { Maximum bandwidth Efficiency } \\
\text { using the above-mentioned roll-off } \\
\text { factors }\end{array}$ & $1.19 \mathrm{bits} / \mathrm{sec} / \mathrm{Hz}$ & $2.57 \mathrm{bits} / \mathrm{sec} / \mathrm{Hz}$ & $3.7 \mathrm{bits} / \mathrm{sec} / \mathrm{Hz}$ \\
\hline Video Codec & MPEG-2 & MPEG-2 & MPEG-4 and programmable \\
\hline
\end{tabular}

DVB-S2 system is capable of supporting several satellite applications, including [36]:

- Broadcast services for SDTV and HDTV;

- Interactive data services and Internet access for consumer applications;

- Professional applications for digital TV contribution and satellite news gathering;

- Backward compatibility with DVB-S, due to the high number of operational DVB-S receivers.

DVB-S2 was designed to fulfil the need for long-awaited spectrum efficiency improvements, which were further augmented by the additional gains of the emerging new video compression technologies, such as H.264/AVC. Additionally, DVB-S2 allows Direct-to-Home broadcasters to launch more SDTV and HDTV broadcast and interactive TV services using the available spectrum resources [35].

The DVB-S2 standard opted for Low Density Parity Check (LDPC) codes [34,37] as the inner channel codes, since they provide a near-Shannon-limit performance at an affordable implementation complexity. The LDPC codes selected use the block lengths of 16200 and 64800 bits with code rates of $1 / 4$, $1 / 3,2 / 5,1 / 2,3 / 5,2 / 3,3 / 4,4 / 5,5 / 6,8 / 9$ and $9 / 10[34,36$, 38]. DVB-S2 uses four modulation schemes, including QPSK, 8PSK, 16APSK and 32APSK, where the APSK schemes mainly target professional applications [36]. The DVB-S2 APSK schemes require a higher SNR for achieving the same performance as the QAM modulations schemes of DVBS2, however they achieve a higher spectral efficiency. The
APSK constellation is formed of uniformly spaced circular centric rings, which exhibit a lower peak-to-mean ratio than the corresponding square-QAM schemes. Hence, they reduce the non-linear distortion effects and the the resultant out-ofband harmonic emissions imposed by high power amplifiers. They are standardised for professional applications, since they require more sophisticated and hence more expensive receivers using non-linear satellite transponders [35].

Table VII compares the possible configurations of DVB-S, DVB-DSNG and DVB-S2. As shown in Table VII, DVB-S2 uses Bose-Chaudhuri-Hocquenghem (BCH) [39] and LDPC as channel codes compared to the RS and convolutional codes for DVB-S and DVB-DSNG. DVB-S2 selected several code rates for the LDPC code in order to allow for a higher flexibility and it is capable of attaining a better performance compared to both DVB-S and DVB-DSNG. Additionally, the DVB-S2 standard was specified to allow a lot of flexibility in configurations including several channel code rates and several possible modulation schemes to use.

DVB-S2 succeeded in providing a bandwidth efficiency gain of up to $30 \%$ over its first-generation counterpart DVB-S. This was made possible by the advances in transmission technology and the availability of new coding as well as modulation schemes. These advances also motivated the production of a second-generation terrestrial broadcast system, namely DVB$\mathrm{T} 2$ [40], in order to increase the capacity over DVB-T and hence to facilitate high-resolution HDTV services or the provision of more broadcast channels in the available terrestrial 
transmission bandwidth.

The key requirements for the development of DVB-T2 were the ability to use the existing domestic receive antennas and the existing transmitter infrastructure, with the intention to support both fixed and hand-held reception. It was also required to provide an improved SFN performance as well as to support both an increased frequency-allocation flexibility and to reduce the Peak-to-Average Power Ratio (PAPR). The reduction of PAPR facilitates the employment of transmitter power amplifiers having a reduced linearity requirement, which in turn results in an increased power efficiency as detailed in [22]. The DVB-T2 standard adopted the same FEC structure as the DVB-S2, in the sense that it uses LDPC as the inner FEC code and a $\mathrm{BCH}$ outer code. Table VI compares the configuration parameters of DVB-T and DVB-T2. As shown in Table VI, DVB-T2 includes more configurations than DVB-T, hence it is a very flexible system with several possible channel code rates, diverse modulation schemes and several pilot patterns, potentially allowing for a possible reduction in the percentage of pilots in the transmitted frame compared to DVB-T. The DVB-T2 system will be detailed in Section VII. According to Table VI, the DVB-T2 system requires less pilots than the DVB-T, hence allowing for a better bandwidth efficiency. Additionally, due to the flexibility of the DVB-T2 system, it can accommodate a bit rate of up to $50.34 \mathrm{Mbit} / \mathrm{sec}$ and a maximum bandwidth efficiency of up to $6.29 \mathrm{bits} / \mathrm{sec} / \mathrm{Hz}$. Table IV shows how to evaluate the bit rate in DVB-T, which is calculated similarly for DVB-T2. Furthermore, it is worth noting that the bit-rate and bandwidth efficiency values presented in Table VI are the values calculated after considering the FEC and taking into consideration the signalling and pilots.

Finally, the second-generation cable TV, namely DVB-C2 system, was ratified, which uses the same FEC and modulation techniques as the DVB-S2 and DVB-T2 standards, in order to form what is referred to as the "family of standards". Similar to DVB-S2 and DVB-T2, the DVB-C2 standard employs BCH and LDPC codes combined with 16-QAM, 64-QAM, 256QAM, 1024-QAM and 4096-QAM as the possible modulation schemes. However, unlike DVB-C, which is a single-carrier system, DVB-C2 employs OFDM. OFDM is used in DVB$\mathrm{C} 2$ for several reasons, including the efficiency and flexibility offered by OFDM, as well as for the sake of allowing DVBS2, DVB-T2 and DVB-C2 to have a similar structure, which simplifies the receiver implementation, hence potentially allowing a single semi-conductor chip to demodulate all the three standards. DVB-C2 uses segmented OFDM, where a receiver with its $8 \mathrm{MHz}$ tuner can extract the specific segment of the broader band containing the required service. The DVB$\mathrm{C} 2$ system is capable of attaining a data rate of up to $60 \%$ higher than that of DVB-C $[41,42]$.

\section{Advanced Television System Committee}

Following the success of the NTSC projects and the demand for a HDTV standard, the Advanced Television System Committee (ATSC) was formed in the USA in order to explore the development of an advanced TV standard. Initially, several standards were proposed based on either analogue or on hybrid analogue-digital techniques, which were all rejected by the
FCC. As a further advance, several all-digital systems were proposed, which led to the development of a system combining the benefits of several of these standards $[6,43]$. Then in 1997, the FCC adopted the ATSC standard and mandated its employment for terrestrial TV broadcast in the USA.

The ATSC employs the MPEG video streaming syntax for the coding of video and the ATSC standard "Digital Audio Compression (AC-3)" for the coding of audio [9,44]. The bit stream is constituted by multiplexed video bit stream packets, audio bit stream packets and data bit stream packets. The structure of these bit streams is also carried as signalling information in the bit stream, which employs the MPEG-2 TS-structure packet format for packetising the video, audio and service information of the different services within the same multiplexed format.

Figure 6 shows a block diagram of the physical layer processing in the ATSC standard. As in the DVB systems, a data randomiser or scrambler is used for randomising the data payload, which results in a flat PSD for using the available spectrum more efficiently. The data randomiser generates the 'XOR' function of all the incoming data bytes with a 16-bit maximum length pseudo random binary sequence, which is initialized at the beginning of each frame. The generator polynomial for the randomiser LFSR used in the ATSC standard is shown in Table VIII. Following the randomiser/scrambler, the data is encoded by a RS encoder, as shown in Figure 6. The RS code used in the ATSC is a $(207,187,10)$ code defined over the GF(256) as shown in Table VIII, with (n$\mathrm{k})=20$ parity bytes attached to the end of the $\mathrm{k}=187$-byte packet. The code is capable of correcting $\mathrm{t}=10$ erroneous bytes. The data is then passed through a convolutional interleaver of depth 52 as shown in Table VIII. The convolutional interleaver interleaves the data and parity bytes, while keeping the first byte in every packet as the sync byte. Again, the sync bytes are used for packet synchronisation in the receiver. As in the DVB standards, an inner convolutional interleaver is used for protecting against burst errors in a fading channel.

The ATSC standards use Trellis Coded Modulation (TCM) [29] after interleaving, which expands the QPSK constellation to 8PSK by absorbing the parity bits without expanding the bandwidth. Hence, 3 bits/symbol are transmitted instead of 2 bits/symbol. The TCM encoder's output is then interleaved by an inner interleaver as shown in Figure 6 and detailed in Table VIII. After the TCM encoder, the signalling information is inserted in order to allow the receiver to synchronise as well as to track the data and hence to facilitate the demodulation of the signal. These include segment sync, frame sync and pilots, as shown in Figure 6. The segment sync is a sync byte that is used for generating a reference clock in the receiver by correlating the received data and finding the location of the known segment sync. On the other hand, the frame sync is an entire packet that is known to the receiver and is used for adapting the ghost-cancelling equaliser. The segment sync and frame sync are BPSK modulated before multiplexing with the TCM data.

The TS packet sync bytes are not trellis-coded, hence replacing the sync bytes by the segment syncs does not have any effect on the data, because the packet sync bytes can be added in the receiver before trellis decoding. The segment 


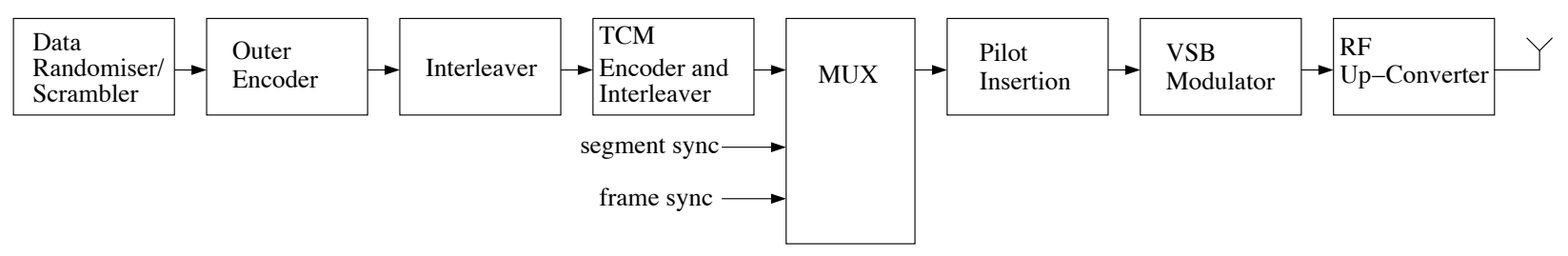

Fig. 6. ATSC transmitter block diagram using the parameters in Table VIII.

TABLE VIII

TRANSMITTER PARAMETERS OF THE ATSC SYSTEM SHOWN IN FIGURE 6.

\begin{tabular}{|l|l|}
\hline Energy dispersal LFSR polynomial & $1+X+X^{3}+X^{6}+X^{7}+X^{11}+X^{12}+X^{13}+X^{16}$ \\
\hline Outer FEC code & $(207,187,10)$ Reed-Solomon code over GF(256) \\
\hline Outer interleaver & Convolutional interleaver with a depth of 52 \\
\hline Inner FEC code & $2 / 3$-rate 4 -state trellis encoder \\
\hline Inner interleaver & 12 groups symbol interleaving \\
\hline Segment sync & used for generating the receiver clock and for recovering the data \\
\hline Frame sync & used to "train" the adaptive ghost-cancelling equalizer in the receiver \\
\hline
\end{tabular}

sync and frame sync are then multiplexed with the trellis coded symbols, followed by incorporating pilots for channel estimation and synchronisation. The VSB modulator removes a large fraction of one of the sidebands, while retaining the other one, followed by the classic RF up-conversion and transmission [45]. More details about the 8-level Vestigial SideBand (8-VSB) trellis coding scheme can be found in $[44]^{4}$.

The 8-VSB modulated ATSC system has a fixed bit rate of $19.39 \mathrm{Mbit} / \mathrm{sec}$ in a $6 \mathrm{MHz}$ channel, after the FEC coding, the pilots and the sync data were added. The ATSC standard does not use OFDM, which makes the equaliser design a real challenge. However, this is not to say that the DVB-T system is better than ATSC; both systems have valid reasons for adopting the specific technology advocated. One of the major benefits of the OFDM modulated systems is that of rendering the radio broadcasts relatively immune to multipath distortion and signal fading. Additionally, OFDM is capable of supporting SFNs, which is not possible for 8-VSB. On the other hand, with the advances in equaliser technology, the attainable 8 -VSB performance in multipath channels is comparable to OFDM, although at the expense of a more complicated equaliser implementation. Furthermore, 8-VSB is nearly a single side-band transmission scheme while OFDM is a double side-band technique, which means that 8-VSB is potentially more bandwidth-efficient than OFDM.

\section{A. $A T S C-M / H$}

Following the increasing popularity of mobile phones and the great demand for wireless multimedia services worldwide, the ATSC produced a new standard for mobile devices, which is known as the ATSC Mobile/Hand-held (ATSC-M/H) standard [46]. The ATSC-M/H system was designed to be fully compatible with the existing ATSC system, where the ATSC$\mathrm{M} / \mathrm{H}$ shares the same transmission scheme as the standard terrestrial ATSC service [44]. This is illustrated in the block diagram of Figure 7, where the channel coding and modulation scheme used in ATSC-M/H is the same as that employed

\footnotetext{
${ }^{4} \mathrm{~A}$ VSB modulator is used for the RF modulation of the 8-level TCM coded signal, hence the terminology 8-VSB. Moreover, the DTV community tends to refer to the ATSC transmitter as the 8-VSB transmitter
}

by the ATSC standard of Figure 6. The ATSC-M/H system uses a specific portion of the available data rate in the ATSC broadcast system, while the rest is still available for the main ATSC service using time-division multiplexing.

Regardless of the system considered, broadcasting over mobile channels is more challenging than over fixed stationary terrestrial radio channels. The $\mathrm{M} / \mathrm{H}$ system allows transmission of the $\mathrm{M} / \mathrm{H}$ data in bursts, which provides some potential power saving for the battery-powered receiver. As shown in Figure 7, the packet multiplexer in the ATSC broadcast system's transmitter receives two sets of input streams: one consisting of the MPEG TS packets of the main ATSC service and another one consisting of the IP-based packets of the M/H service data [46]. As shown in Figure 7, the transmitter multiplexes the ATSC MPEG TS and ATSC-M/H IP-based stream, before passing it to the channel coding and modulation blocks of Figure 7. The ATSC-M/H information is comprised of the IP transport stream and the $\mathrm{M} / \mathrm{H}$ structure data, which includes the Transmission Parameter Channel (TPC) Data, such as the sub-frame index and slot index as well as the Fast Information Channel (FIC). The FIC carries cross-layer information for enabling a fast $\mathrm{M} / \mathrm{H}$ service acquisition [46]. For compatibility with legacy 8 -VSB receivers, the ATSC-M/H service data is encapsulated in specifically designed MPEG transport stream packets, designated as $\mathrm{M} / \mathrm{H}$ Encapsulation (MHE) packets [46].

The M/H system's signalling information includes information about the structure of the main service as well as the $\mathrm{M} / \mathrm{H}$ service data in the transmitted frame. The data is structured in $\mathrm{M} / \mathrm{H}$ frames, which include data from the main and $\mathrm{M} / \mathrm{H}$ services. The $\mathrm{M} / \mathrm{H}$ data is partitioned into ensembles, which might contain one or more services. Each ensemble uses an independent RS frame and can be protected by a different code rate, depending on the specific application. Each $\mathrm{M} / \mathrm{H}$ frame is divided into 5 consecutive sub-frames, with each sub-frame consisting of 16 consecutive $\mathrm{M} / \mathrm{H}$ slots, where an $\mathrm{M} / \mathrm{H}$ slot is the basic time period for the multiplexing of the $\mathrm{M} / \mathrm{H}$ data and main service data [46]. A slot consists of 156 packets, which may carry only legacy TS-packets or may carry a M/H-group of 118 MHE packets and 38 legacy TS packets. Additionally, the ATSC-M/H standard [46] defines an $\mathrm{M} / \mathrm{H}$ parade, which is 


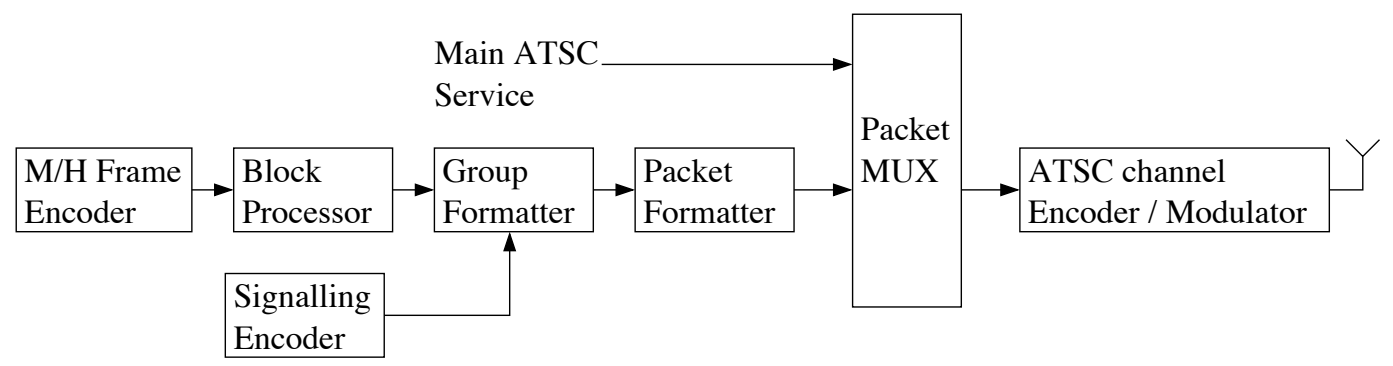

Fig. 7. ATSC broadcast system with TS Main and Mobile/Hand-held $(\mathrm{M} / \mathrm{H})$ services. The channel coding and modulation as identical to those shown in Figure 6 using the parameters in Table VIII. The M/H frame encoder is shown in details in Figure 8, the block processor is detailed in Figure 9 and the signalling encoder is shown in Figure 10. The system parameters are listed in Table IX.

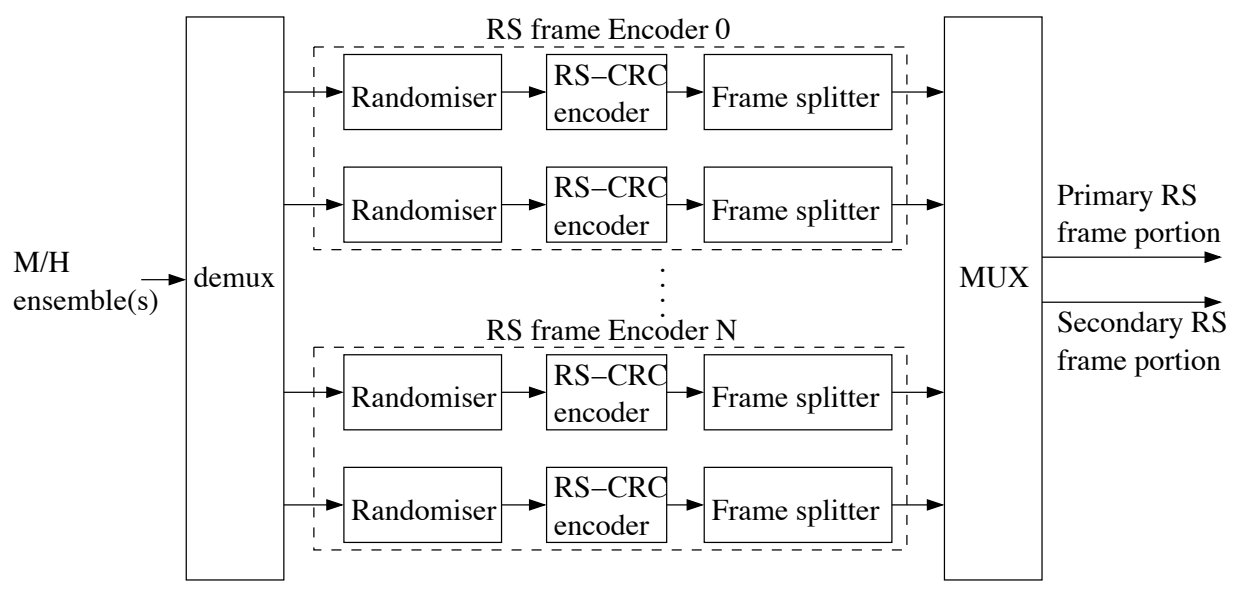

Fig. 8. The ATSC-M/H frame encoder used in Figure 7 and using the parameters detailed in Table IX.

a collection of M/H-groups. An M/H Parade carries data from one or two particular RS Frames depending on the RS Frame mode. The RS Frame is a packet-level FEC structure for the $\mathrm{M} / \mathrm{H}$ data. Each RS Frame carries, and FEC encodes, an M/H Ensemble, which is a collection of $\mathrm{M} / \mathrm{H}$ services providing the same quality of service. The ATSC-M/H accommodates some extra training symbols and extra FEC is incorporated, as shown in Figure 7. According to Figure 7, this includes the $\mathrm{M} / \mathrm{H}$ frame encoder of Figure 8, the block processor of Figure 9 and the signalling encoder of Figure 10.

According to Figure 8, the demultiplexer separates the input ensemble and routes them into their corresponding RS frame encoder. The number of RS frame encoders is the same as the number of $\mathrm{M} / \mathrm{H}$ parades. The RS frame encoder operates in two modes: the first mode produces a single RS frame, normally the primary RS frame in Figure 8 and the other mode produces two frames known as primary and secondary RS frames. The primary and secondary RS frame portions are transmitted in different $\mathrm{M} / \mathrm{H}$ groups.

As shown in Figure 8, separate randomisers are used for the primary and secondary RS frames and also different randomisers are employed for different parades. The $\mathrm{M} / \mathrm{H}$ randomiser LFSR polynomial is shown in Table IX. After the randomisation/scrambling process, a RS-Cyclic Redundancy Check (CRC) encoder is employed, which RS-encodes the data followed by concatenating a CRC syndrome check sequence. As shown in Table IX, there are three modes for the RS encoder and each add a different number of parity bytes. Then, after the RS-CRC encoding, a frame splitter is used in

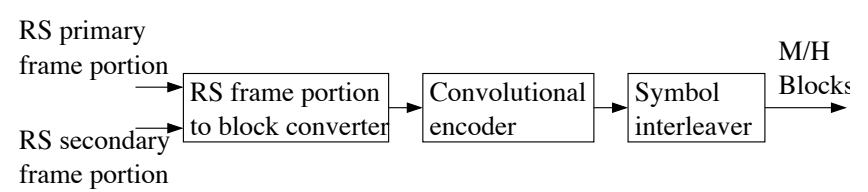

Fig. 9. The ATSC-M/H block processor used in Figure 7 and using the parameters detailed in Table IX.

order to maintain the correct timing of the output data received from the frame encoder.

According to Figure 7, a block processor is employed after the frame encoder. A block diagram of the block processor is shown in Figure 9. The main function of the block processor is to incorporate an improved error correction capability by using a convolutional code and an interleaver. Before any FEC is employed, the output of the frame encoder, which incorporates the RS primary and secondary segments is then organised into a serial frame. This is carried out according to a defined framing structure as detailed in [46]. The convolutional code used is a punctured $1 / 2$-rate or $1 / 4$-rate code, as shown in Table IX. After the convolutional encoder, a block interleaver is employed in order to scramble the output of the encoder.

The ATSC-M/H signalling information, which constitutes vital side-information, is also FEC-coded in order to protect the signalling information. The block diagram of the signalling encoder is shown in Figure 10. The signalling information includes the TPC and FIC. The TPC signals the M/H transmission parameters, including the various FEC modes and 
TABLE IX

TRANSMITTER PARAMETERS OF THE ATSC-M/H SYSTEM SHOWN IN FIgURES 7, 8, 9, 10.

\begin{tabular}{|l|l|}
\hline M/H randomiser LFSR polynomial & $1+X+X^{3}+X^{6}+X^{7}+X^{11}+X^{12}+X^{13}+X^{16}$ \\
\hline Frame encoder RS code mode 0 & $(211,187,12)$ Reed-Solomon code over GF(256) \\
\hline Frame encoder RS code mode 1 & $(223,187,12)$ Reed-Solomon code over GF(256) \\
\hline Frame encoder RS code mode 2 & $(235,187,12)$ Reed-Solomon code over GF(256) \\
\hline Block processor conv. code rates & $1 / 2,1 / 4$ \\
\hline TPC RS code & $(18,10,4)$ Reed-Solomon code over GF(256) \\
\hline FIC RS code & $(51,37,7)$ Reed-Solomon code over GF(256) \\
\hline Signalling encoder inner FEC code & $1 / 4$-rate parallel concatenated convolutional code \\
\hline
\end{tabular}

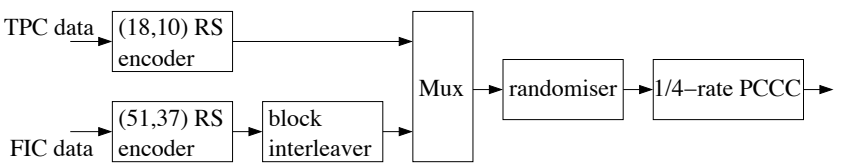

Fig. 10. The ATSC-M/H signalling encoder used in Figure 7 and using the parameters detailed in Table IX.

the $\mathrm{M} / \mathrm{H}$ framing information. On the other hand, the FIC contains cross-layer information used for allowing a faster acquisition. The TPC data is encoded by a $(18,10,4)$ RS code over $\mathrm{GF}(256)$, while the FIC data is encoded by a $(51,37,7)$ RS code over GF(256), as shown in Table IX, followed by a block row-column interleaver. The TPC and FIC data are then multiplexed before passing them through a randomiser, as shown in Figure 10. The randomiser is followed by a $1 / 4$-rate Parallel Concatenated Convolutional Code (PCCC), as shown in Table IX, where the PCCC operates on frames of length 552 bits [46]. The $\mathrm{M} / \mathrm{H}$ data and signalling data are then multiplexed according to a specific framing structure and then the resultant frame is multiplexed with the main ATSC service data, before passing it through the ATSC channel encoder and modulator of Figure 6.

Recently, ATSC's Planning Team 2 was charged with exploring options for a Next Generation Broadcast Television (NGBT) system, which is referred to as "ATSC 3.0", with the mission of investigating candidate technologies and services, whilst eliminating the requirement of conceiving a system which is backward compatible with the current ATSC system. A report has been produced in September 2011 [47], which presents some high level conclusions regarding the potential candidate technologies, content suggestions and general development timing, whilst allowing collaboration with other standardisation bodies, such as the DVB.

\section{B. J.83-B Cable Standard}

The digital cable broadcast began in the early 1990s in North America [48]. The North American cable standard is specified in the International Telecommunications Union's (ITU) ITU-T Recommendation J.83, Annex B [49]. Figure 11 shows the transmitter block diagram of the J.83-B standard, which was designed for transmission over the cable channel. Again, the cable channel is a bandwidth-limited channel and suffers from white noise, interference and multipath distortion as mentioned in Section III.

As shown in Figure 11, J.83-B uses a concatenation of modules that are used in all DTV standards, including a randomiser, an outer encoder, an inner encoder, interleavers

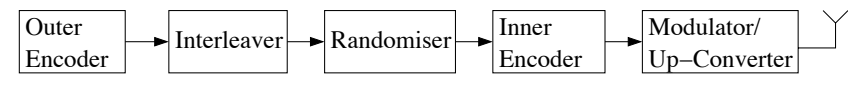

Fig. 11. J.83-B transmitter block diagram using the parameters shown in Table X.

and a modulator. The MPEG TS is first RS-encoded by a $(128,122,3)$ RS code defined over GF(128), which has the capability to correct 3 7-bit symbol errors per RS-block. Following the RS encoder, convolutional interleaving is performed for the sake of eliminating burst errors. The J.83-B standard defines several possibilities for the depth of the convolutional interleaver, as shown in Table X. Then a randomiser is used in order to provide a flat PSD for the constellation symbols using the randomisation polynomial shown in Table X [49]. Afterwards, the bits are encoded and modulated using either 16 -state rate-5/6 64-QAM TCM or 16 -state rate-7/8 256QAM TCM. Further details about the TCM used in the J.83-B standard can be found in [49]. Afterwards, the data is upconverted and transmitted.

\section{Integrated Services Digital BroadCAsting}

In Japan, the Japanese public broadcaster NHK had a vision for digital broadcasting in the 21st century for the transmission of HDTV using digital technology. This vision dates back to the 1980s and aimed for converting all broadcasting systems to digital technology in order to accommodate high-quality video/audio and multimedia services, while allowing users to enjoy a high-quality of service both in the home and on the move $[14,50]$.

\section{A. $I S D B-T$}

The Association of Radio Industries and Businesses (ARIB) in Japan approved the specifications for a digital terrestrial broadcasting system termed as Terrestrial Integrated Services Digital Broadcasting, or ISDB-T for short, in 1998.

Figure 12 shows the ISDB-T transmitter block diagram, which includes the same basic blocks as the other standards, including scrambling/energy dispersal, interleaving, channel coding and modulation. The ISDB-T system uses MPEG-2 Video coding and MPEG-2 advanced audio coding (AAC) and it adopts MPEG-2 TS for encapsulating the data stream.

The ISDB-T system is an OFDM-based system but it uses Band-Segmented Transmission OFDM (BST-OFDM). As the name suggests, BST-OFDM divides the available bandwidth into basic frequency blocks called segments [4]. The BSTOFDM improved on the Coded-OFDM by modulating some 
TABLE $X$

TRANSMITTER PARAMETERS OF THE J.83-B SYSTEM SHOWN IN FIgURE 11.

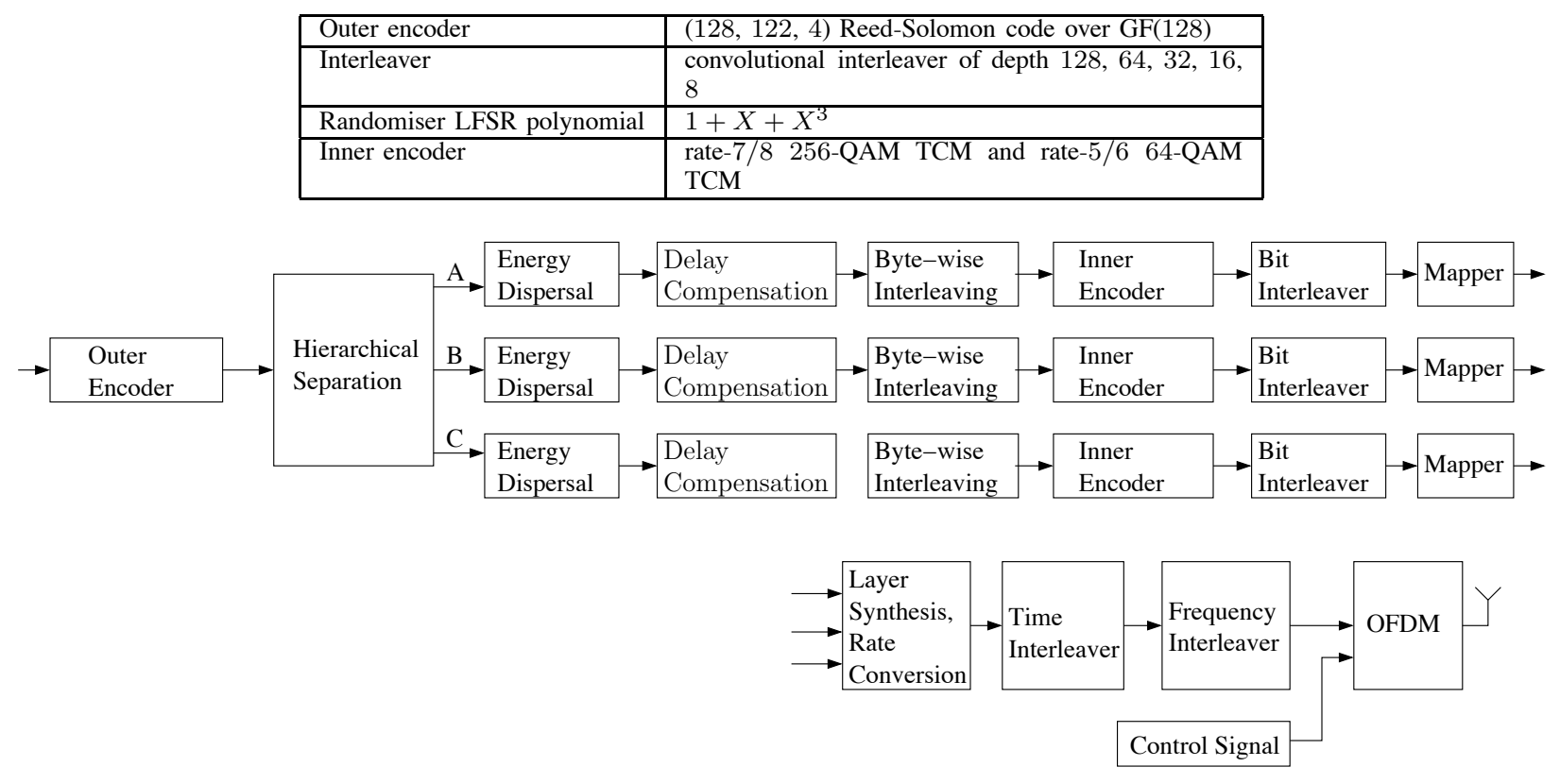

Fig. 12. ISDB-T transmitter block diagram using the parameters listed in Table XI.

TABLE XI

TRANSMITTER PARAMETERS OF THE ISDB-T S YSTEM SHOWN IN FIGURE 12.

\begin{tabular}{|l|l|}
\hline Outer encoder & $(204,188,8)$ Reed-Solomon code over GF(256) \\
\hline Randomiser LFSR polynomial & $1+X^{14}+X^{15}$ \\
\hline Byte-wise interleaver & convolutional interleaver of depth 12 \\
\hline Inner encoder & convolutional code with code rates of $1 / 2,2 / 3,3 / 4$, \\
& $5 / 6,7 / 8$ \\
\hline Bit interleaver & serial-to-parallel conversion followed by bit delay. \\
& The number of parallel streams is different for dif- \\
& ferent constellation sizes. \\
\hline Modulation schemes & QPSK, 16-QAM, 64-QAM \\
\hline
\end{tabular}

OFDM carriers differently from others within the same multiplex. In ISDB-T, BST-OFDM relies on 13 segments, where different segments can employ different coding-modulation schemes.

A fundamental characteristic of BST-OFDM is the ability to use different modulation and coding parameters in one or more OFDM segments. This leads to the idea of hierarchical transmission. ISDB-T uses hierarchical transmission, where the transmission parameters including the modulation scheme of the OFDM carriers, the coding rate of the inner code and the time interleaver length can be independently specified for each OFDM data segment. The ISDB-T defines a maximum of three layers or three different segment groups, known as layers A, $\mathrm{B}$ and $\mathrm{C}$, for transmission in a channel at the same time. More explicitly, the video-encoded bits typically have different errorsensitivity, since their corruption results in different perceptual effects. Hence the most sensitive bits are typically encoded by stronger FEC codes and may be mapped to less error-sensitive modulation schemes. It is possible to allow for the reception of a single layer; this is normally known as partial reception and it is specified in order to allow for a low-rate hand-held mobile receiver to demodulate the signal or to allow the detection of the most important video bits such as for example the motion-vector encoding bits, even if the less important bits were corrupted.

The MPEG-2 TS is first encoded by a RS $(204,188,8)$ code over GF(256), as shown in Figure 12 and Table XI. The output of the RS encoder is then divided into 204-byte packets and then sorted into layers, namely into up to three layers according to the pre-determined control information, as shown in Figure 12. In the case of non-hierarchical transmission, a single stream is defined for transmission. The processing within every layer is carried out in the same way, although the configuration of the different blocks in the different layers might be different.

The data in every layer is then randomised by the scrambler in Figure 12 for energy dispersal using the LFSR polynomial shown in Table XI. The randomised stream is then passed through a delay-compensation block, as shown in Figure 12. The delay-compensation is used in order to compensate for the difference in the delay between the separate streams [50]. More explicitly, the delay caused by the byte-wise interleaving is different in the different streams, due to the difference in the modulation and coding scheme employed in each layer; hence, in order to compensate for the delay differences, a delay-compensation is performed prior to the interleaving and this includes compensation for the delay difference in the deinterleaving process as well. 


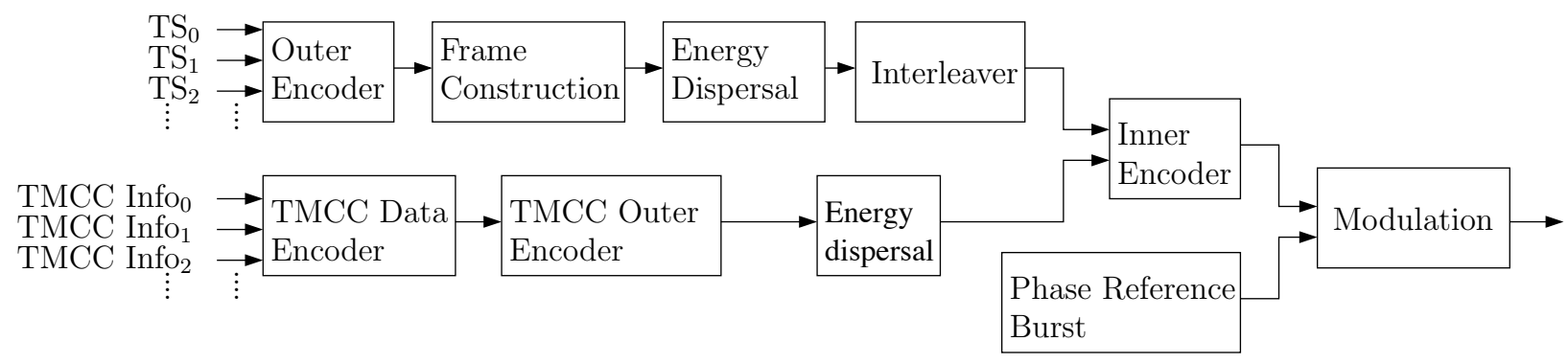

Fig. 13. ISDB-S transmitter block diagram using the parameters in Table XII.

The data is then byte-wise interleaved using a convolutional interleaver of depth 12, as shown in Figure 12 and Table XI. Following the interleaver, the data is convolutionally encoded, where different code rates can be used in any of the three layers. The convolutional codes use a $1 / 2$-rate mother code and puncturing, resulting in code rates of $1 / 2,2 / 3,3 / 4,5 / 6$ and $7 / 8$, as shown in Table XI. The data is then bit-interleaved and then mapped to QPSK, 16-QAM or 64-QAM symbols. As mentioned in Table XI, the bit interleaver first converts the serial input stream to parallel streams, where the number of parallel streams is equal to the number of bits per constellation symbol. Then, following the serial-to-parallel conversion, bitdelay is applied to the different streams, before mapping the bits to the corresponding constellation points.

As shown in Figure 12, the output of the three layers in the ISDB-T transmitter is passed to a layer-synthesis and rate conversion module. The goal of this is to assign the output of each layer to its corresponding segment in the BST-OFDM scheme. Afterwards, the data stream is time-interleaved, followed by a frequency interleaver in order to improve mobile reception and to counteract the effects of multi-path fading. The frequency interleaving is designed in a way to allow for the separation of the segments independently from the other segments in the transmitted BST-OFDM signal. This facilitates partial reception using a narrow bandwidth low-rate mobile receiver. Finally, the data stream is combined with the control signal to form an OFDM symbol before transmission, as shown in Figure 12. The control signal includes the layerconfiguration in hierarchical transmission, including the side information required for detecting the different layers and the associated partial reception information.

In summary, the ISDB-T system uses BST-OFDM and supports the hierarchical transmissions of up to three layers, usually known as layers A, B and C, as shown in Figure 12. The transmission parameters can be changed in each of these layers with the aim of allowing a low-complexity hand-held receiver to demodulate a single segment of the hierarchical transmission [51]. This would not necessarily be perceptually objectionable, since the small hand-held screen does not require as high a resolution as in HDTV screens.

\section{B. $I S D B-S$}

On the other hand, the satellite digital broadcasting of HDTV in Japan employs the ISDB-S standard [52,53]. With a rainy environment in mind, as in Japan, the ISDB-S was designed to be a flexible system capable of mitigating the effect of rain attenuation. The ISDB-S was designed to cover a wide range of requirements, including high-throughput HDTV transmissions and high reliability data services [52]. ISDB$\mathrm{S}$ facilitates flexible transmission configuration in accordance with the broadcasters' specific service and content by using the so-called Transmission and Multiplexing Configuration Control (TMCC) information. Figure 13 shows the block diagram of the ISDB-S transmitter, which generates three main types of signals:

1) Main signal that carries the MPEG TS programme content.

2) TMCC signal that includes control information, such as the modulation and code rate as well as the identification of the services' TSs.

3) Burst signal used for ensuring stable carrier recovery at the receiver.

As shown in Figure 13, multiple MPEG TSs are combined into a single stream that is processed in the channel coding pipeline. The control data that carries information for every TS packet is encoded into the TMCC signal that is also channel coded before combining with the main data TS for inner encoding and modulation. The data and control information are combined into a single sequence using time division multiplexing according to a pre-defined sequence. Then the inner-encoded stream is time division multiplexed with the burst data before modulation and transmission, as shown in Figure 13.

As shown in Figure 13, the TS packets are encoded in an outer $(204,188,8)$ RS-encoder defined over GF(256). The output of the RS encoder is passed through a frame construction process, where a data frame is formed from the encoded packets. This is carried out in a way to consider the variation in the spectral efficiency of different modulation and inner code combinations. The data frame is then randomised for energy dispersal/scrambling using the polynomial given in Table XII. An interleaver follows the energy dispersal stage, as shown in Figure 13, where inter-frame interleaving is performed using a row-column-like interleaver.

The TMCC carries the specific modulation/coding combination used for each slot, the MPEG-TS identification in every slot and some further control information [52]. The TMCC is normally transmitted before its corresponding data, since it carries the side-information necessary for the correct decoding of the data. The information carried by the TMCC signal is formatted in the TMCC data encoder of Figure 13 [52]. The TMCC data is then encoded by a high-rate $(64,48,8)$ 
TABLE XII

TRANSMITTER PARAMETERS OF THE ISDB-S SYSTEM SHOWN IN FIGURE 13.

\begin{tabular}{|l|l|}
\hline TS outer encoder & $(204,188,8)$ Reed-Solomon code over GF(256) \\
\hline Data stream randomiser LFSR polynomial & $1+X^{14}+X^{15}$ \\
\hline TMCC outer encoder & $(64,48,8)$ Reed-Solomon code over GF(256) \\
\hline TMCC stream randomiser LFSR polynomial & $1+X^{14}+X^{15}$ \\
\hline Inner encoder & convolutional code with code rates $1 / 2,2 / 3,3 / 4$, \\
& $5 / 6,7 / 8$ \\
\hline Modulation schemes & BPSK, QPSK, 8 -PSK \\
\hline Modulation/coding possible combinations & $2 / 3$-code TCM 8 -PSK \\
& $1 / 2,2 / 3,3 / 4,5 / 6,7 / 8$ rate code with QPSK \\
& $1 / 2$ rate code with BPSK \\
\hline
\end{tabular}

RS encoder, followed by energy dispersal using the same polynomial as the data stream, as shown in Table XII. The main signal and the TMCC signal are then time-division multiplexed in every frame according to the modulation/code combination for every slot.

The time-multiplexed frame is then channel coded and modulated. The ISDB-S uses an inner convolutional encoder having a 1/2-rate 128-state mother code and specifies the following possible combinations of the modulation/coding schemes, as shown in Table XII:

1) $2 / 3$ rate TCM with 8 -PSK;

2) $1 / 2,2 / 3,3 / 4,5 / 6,7 / 8$ rate convolutional code with QPSK;

3) 1/2-rate convolutional code combined with BPSK.

On the other hand, due to the fact that multiple transmission configurations could be used combined in the ISDB-S scheme and given the fact that optimal carrier-recovery differs per modulation, a BPSK-modulated burst is added and timemultiplexed with the main video signal in order to maintain stable carrier recovery. These bursts are inserted at specified instants known to the receiver. Furthermore, these bursts might be inserted at the beginning of the frame for attaining frame synchronisation at the receiver.

\section{International System for Digital Television}

International System for Digital Television, or ISDTV for short, is the DTV system developed in Brazil and used in several countries in South America. It is also known Sistema Brasileiro de Televisao Digital (SBTVD) and ISDB-T Brazilian Version (ISDB-Tb). The transmission technology used in ISDTV is identical to that used in the ISDB-T [4].

The basic difference between ISDTV and the ISDB-T is the use of H.264 video coding compared to MPEG-2 that is used in the ISDB-T. Additionally, ISDTV introduced an interactive middleware platform known as Ginga. Furthermore, ISDTV specified using WiMAX as the standard for the return channel in interactive services [4].

\section{Vi. Digital Terrestrial Multimedia Broadcast}

In China, the development of digital TV started in 1994 when the Chinese government issued a research initiative. In 2006, the "Standard for Radio Film and Television Industry in China" announced the release of the DTV standard, known as Digital Terrestrial Multimedia Broadcast (DTMB) and referred to as GB20600-2006, after extensive lab and field tests [54, 55]. Compliance with this standard was made mandatory by
August 2007 and it is expected that the Analogue TV will be switched off by 2015 .

Figure 14 shows a high level block diagram of the DTMB transmitter using the parameters listed in Table XIII. The physical layer transmission system defines Physical Logical Channels (PLCH), whose throughput and robustness are configured according to the channel coding rate, modulation constellation and the number of time slots allocated. The PLCH is divided into 40 time slots with one Control Logical Channel (CLCH) and up to 39 Service Logical Channels (SLCH). The CLCH occupies the first time slot in the PLCH, while a SLCH may occupy several time slots to provide different transmission capacities for different broadcast services. The PLCH is multiplexed in MPEG-2 TS structure [56].

As shown in Figure 14, the MPEG TS is first randomised for energy dispersal/scrambling using the LFSR polynomial shown in Table XIII. The randomised data stream is then encoded using a variable-rate $(240, K)$ RS encoding over $\mathrm{GF}(256)$, where $K$ can have the following values 240,224 , 192 and 176. After RS encoding, the data is byte-interleaved using a row-column interleaver. The output of the outer encoder is grouped into bytes, where a row is formed by an RS output packet of 240 bytes and the number of rows is dependent on the specific combination of the modulation scheme and the inner encoder code rate. The data is written row-wise and read column-wise [54].

The interleaved frame is then encoded by an LDPC encoder having a code rate of $1 / 2$ or $3 / 4$. The output frame of the LDPC encoder has a length of 9216 bits, which implies that the input is 4608 bits long for the $1 / 2$-rate code and 6912 bits for the 3/4-rate code. The output frame of the LDPC encoder is then bit interleaved. The bit interleaver used in Figure 14 is a row-column bit interleaver, which operates on the LDPC output frame. The bit-interleaved bit stream is then modulated using BPSK, QPSK or 16-QAM constellations.

As shown in Figure 14, the data stream is then multiplexed with the scattered and continual pilots. The pilots are known to the receiver and are used for carrier acquisition, clock synchronisation and channel estimation. The output frame is then scrambled by a complex pseudo-random sequence in order to achieve faster synchronisation and more accurate channel estimation at the receiver. Afterwards, the scrambled sequence is OFDM modulated using FFT sizes of 1024 or 4096 symbols.

The DTMB standard was designed to support fixed as well as mobile receivers; hence, it was designed as a flexible system capable of broadcasting a number of HDTV programs 


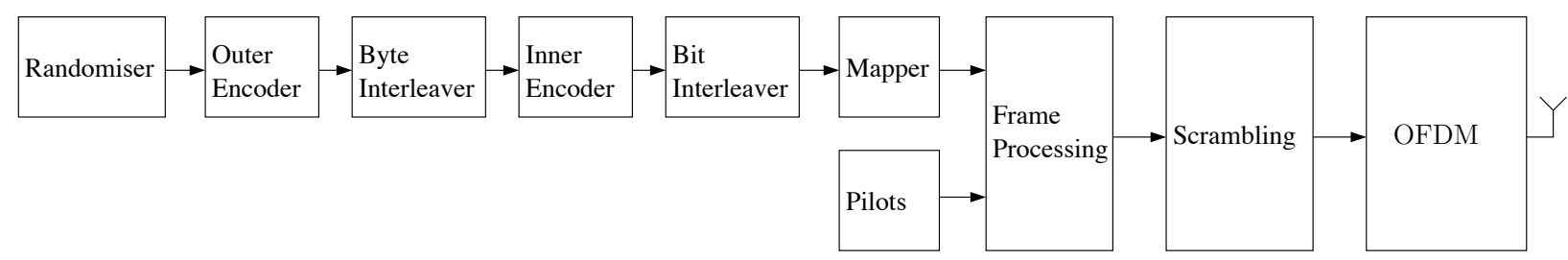

Fig. 14. DTMB transmitter block diagram using the parameters shown in Table XIII.

TABLE XIII

TRANSMITTER PARAMETERS OF THE DTMB SYSTEM SHOWN IN FigURE 14.

\begin{tabular}{|l|l|}
\hline Randomiser LFSR polynomial & $1+X^{2}+X^{3}+X^{4}+X^{8}$ \\
\hline Outer encoder & $(240, K)$ Reed-Solomon code with $K=240,224$, \\
& 192 or $176, \mathrm{GF}(256)$ \\
\hline Byte interleaver & row-column byte interleaver \\
\hline Inner encoder & LDPC code with code rates $1 / 2$ and $3 / 4$ \\
\hline Bit interleaver & row-column bit interleaver \\
\hline Modulation schemes & BPSK, QPSK, 16-QAM \\
\hline
\end{tabular}

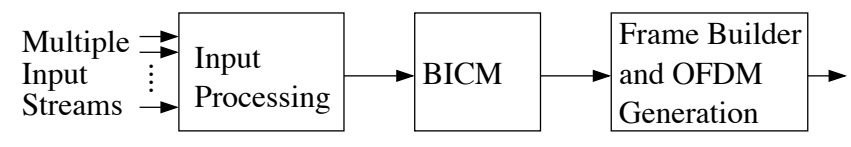

Fig. 15. DVB-T2 transmitter block diagram. The Input Processing block is detailed in Figure 16 and the BICM block is detailed in Figure 17.

combined with several SDTV and other multimedia content. It supports FEC-coded data rates in the range of $4.8 \mathrm{Mbit} / \mathrm{sec}$ to $32.5 \mathrm{Mbit} / \mathrm{sec}$ and it also supports both single as well as multiple frequency networks.

\section{Design EXAMPle: DVB-T2 System ARCHITECTURE}

In Sections III-VI we provided an overview of the transmission techniques used in the physical layer of the different DTV broadcasting standards. In this section we will provide a more detailed portrayal of the DVB-T2 physical layer architecture. We have selected DVB-T2 as our design example, since it is the most recent DTV broadcasting standard and hence it represents the family of state-of-the-art techniques used in DTV. Additionally, there is a paucity of information on DVB$\mathrm{T} 2$ in the literature.

As discussed in Section III in this paper, in order to meet the demand of TV viewers for video-on-demand, HDTV and mobile TV, DVB-T2 was specified as the second generation terrestrial TV standard. Constrained by limited bandwidth availability, the terrestrial TV platform needed a more efficient transmission system to meet the demands of the future and to allow for the launch of new services. The DVB Project responded in June 2008 with the publication of the DVB-T2 specification [40].

The DVB-T2 standard was specified according to the following commercial requirements: re-use of the existing rooftop antennas with a minimum of $30 \%$ increase in the capacity compared to DVB-T, allow reception in both fixed as well as mobile environments, offer a high grade of flexibility and provide a mechanism for reducing the peak-to-average power ratio [57].
Figure 15 shows the transmitter block diagram of the DVBT2 standard, where the input processing block will be detailed in the context of Figure 16, followed by a Bit-Interleaved Coded Modulation (BICM) block shown in Figure 17, a frame builder and OFDM modulation process. The input to the DVBT2 transmitter can be one or more MPEG TS and/or one or more Generic Streams (GS) [19]. The Generic Stream Encapsulation (GSE) was defined by the DVB project as a means for efficient Internet Protocal (IP) based transmission technique, which is also capable of transmitting other networklayer packets over a "generic" physical layer [19]. The TS and/or GSE packets can be pre-processed for de-multiplexing or separating multiple services. This data is then carried in individual streams, which are referred to as Physical Layer Pipes (PLP) in DVB-T2. The concept of PLPs was conceived in order to allow for a service-specific robustness with different input stream types. More explicitly, the different PLPs can carry different input stream types, such as TS or GSE, and these can employ different coding and modulation schemes. The PLP allows the transport of data, regardless of its structure with PLP-specific physical layer configuration, including the coding and modulation schemes and interleaving depth.

As shown in Figure 15, the DVB-T2 transmitter input can be one or more PLP streams. DVB-T2 standard defines two modes of operation:

- Mode A, when a single PLP is transmitted;

- Mode B, when multiple PLPs are transmitted.

Additionally, when more than one PLP is transmitted, there could be a common PLP per group of PLPs. The common PLP contains data shared by all the PLPs in the group, hence eliminating the need to duplicate the transmission of this common data for every PLP. The PLPs that contain data symbols are known as data PLPs. In mode B, a group of data PLPs can have a common PLP. The common PLP is formed for the scenario, when there is common data shared by several data PLPs. This means that it is not compulsory to have a common PLP per group of data PLPs. On the other hand, there could be more than one common PLP with one common PLP per group of data PLPs. In mode A, there is a single PLP, which is a data PLP and there is no common PLP 
transmitted. The receiver is expected to demodulate only one PLP and its corresponding common PLP, if there is one.

Figure 16 shows the block diagram of the Input Processing stage of Figure 15. The input processing is performed per PLP at the transmitter and it is constituted of an input synchroniser, null-packet deletion, CRC encoder, Base-Band (BB) header insertion, scheduling and scrambling. The data processing in the DVB-T2 modulator is not guaranteed to generate constant bit-rate stream; hence, a stream synchroniser in employed in the Input Processing block of Figure 15 and 16 in order to output a constant bit-rate stream having a constant end-toend transmission delay for any input data format. On the other hand, in mode B the input of the transmitter is delayed appropriately when there is a common PLP for a group of data PLPs, in order to allow for transport stream recombining of the data PLP and its corresponding common PLP in the receiver without requiring additional memory.

Furthermore, it is required to have the same bit rate at the output of the transmitter and at the input of the receiver with a constant end-to-end delay. However, for some TS services, there could be null-packets to accommodate a variable bitrate service in a constant bit-rate stream. Therefore, in order to avoid transmission of un-necessary data, these null packets are identified and removed in the null packet deletion module of Figure 16. The null packets' information is passed to the receiver as part of the signalling information in order to allow the receiver to re-construct the transmitted stream. After the null-packet deletion, a CRC sequence is attached in order to allow for error detection at the packet level, where the CRC encoder polynomial is shown in Table XIV. Then, a header is inserted at the beginning of the frame in order to describe the format of the data field. This is described in detail in [40].

As shown in Figure 16, the header insertion is followed by a scheduler and signalling block. This block does not change the data stream in the PLPs, it rather decides as to which PLP data is carried in which OFDM symbol and hence generates the required side-information signalling information about the output frame structure. Hence, the "scheduler and signalling" block of Figure 16 generates signalling information, without modifying the data stream, that is used in the OFDM modulation module and then passed to the receiver for facilitating the correct demodulation of the transmitted signal. Following the scheduler, each PLP's data stream is scrambled for energy dispersal and randomisation. The scrambler polynomial is given in Table XIV.

Having described the input processing for mode B case, let us now consider mode A. In mode A there is only a single stream relying on the same processing, shown in Figure 16, except for the scheduling, which in this case is simply a oneto-one mapping of the PLP data stream to the output frame structure.

As described above, the input processing of Figure 16 used in DVB-T2 is performed on a per PLP basis. Similarly, the BICM is performed separately for the different PLPs, as shown in Figure 17. After input processing of every PLP, as detailed in Figure 15, a frame is passed to the BICM stage of every PLP. The frame is encoded in an outer encoder followed by an inner encoder, as seen in Figure 17. The outer encoder used in DVB-T2 is a BCH $\left(K_{B C H}, N_{B C H}\right)$ code [29] as shown in
Table XIV. The input to the binary $\mathrm{BCH}$ encoder is a set of $K_{B C H}$ bits and its output is comprised of $N_{B C H}$ bits, known as a BBFrame.

The BBFrames output of the $\mathrm{BCH}$ encoder is passed to an inner encoder, as shown in Figure 17. The output of the inner encoder is referred to as "FECFrame". The DVB-T2 standard uses LDPC codes as the inner code, whose output is known as FECFrame. DVB-T2 uses two FECFrame lengths. The short frame length is 16200 bits, while the normal frame length is 64800 bits. Furthermore, DVB-T2 uses the following LDPC code rates: $1 / 4^{5}, 1 / 2,3 / 5,2 / 3,3 / 4,4 / 5,5 / 6$. The values of $K_{B C H}$ and $N_{B C H}$ are listed in Tables XV and XVI for the normal and short frames, respectively.

After the inner LDPC encoder of Figure 17, the data is bit interleaved, which consists of parity interleaving followed by a row-column interleaving stage with a column twist. The LDPC code used in DVB-T2 provides non-uniform error-protection levels for each bit and this depends on the column-weight of the parity check matrix. This means that the protection level of bits in the same constellation point of a multi-level modulation is not uniform. Hence, the bit interleaver of Figure 17 was designed to control the mapping of the FEC coded bits to the constellation bits. The parity interleaving is introduced as a new feature in DVB-T2, where in the absence of the parity interleaving, many constellation symbols would contain coded bits which connect to the same check node and this will degrade the system performance, especially in channels imposing packet erasures. Hence, the parity interleaving part of the bit interleaving was designed to eliminate any performance degradation in channels inflicting packet erasures. After parity interleaving, the data is written in twisted column-wise and read row-wise. Twisted column-wise writing is performed by writing to row 'a' in column 'A' followed by writing to row ' $b$ ' in column ' $A+1$ ', where $a$ and $b$ may be different.

Similarly, for the sake of avoiding the undesirable interaction between the bits carried in a constellation point and the LDPC code structure, a de-multiplexer is used in addition to the bit interleaver in Figure 17. The output of the bit interleaver is de-multiplexed in order to output a stream of bits, which is then mapped to the constellation points. The de-multiplexing is defined in detail in [40]. The output of the de-multiplexer is then mapped to constellation cells ${ }^{6}$, as shown in Figure 17. DVB-T2 uses QPSK, 16-QAM, 64-QAM and 256-QAM as the optional modulation schemes, as detailed in Table XIV.

DVB-T2 also allows the employment of rotated constellations [58] for the sake of improving the achievable system performance in frequency-selective channels imposing packet erasures. It operates by rotating a QAM constellation by a specific angle and then cyclically rotating the I- and Qcomponents of the frame before time- and frequency-domain interleaving. The objective of these operations is that when the I- and Q-components are combined in the receiver after the de-interleaving stage, they would have been affected independently by the frequency selectivity of the channel and hence

\footnotetext{
${ }^{5}$ This code rate is used for the signalling side-information relying on the short frame length of 16200 bits frame, but not for data frames.

${ }^{6}$ DVB-T2 refers to a constellation point as a cell instead of symbol in order to avoid any confusion with OFDM symbols.
} 


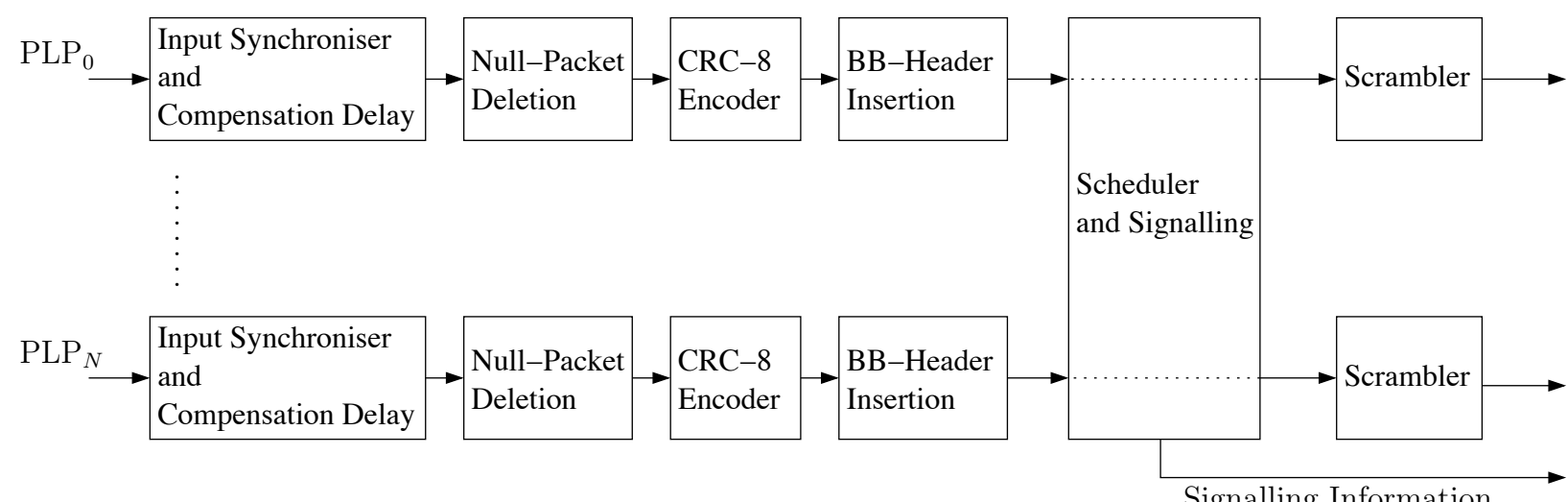

Signalling Information

Fig. 16. Block diagram of the DVB-T2 Input Processing block shown in Figure 15 using the system parameters shown in Table XIV.

TABLE XIY

TRANSMITTER PARAMETERS OF THE DVB-T2 SYSTEM SHOWN IN FIgURES 15, 16, 17.

\begin{tabular}{|l|l|}
\hline CRC-8 encoder polynomial & $1+X^{2}+X^{4}+X^{6}+X^{8} 7+X^{8}$ \\
\hline Scrambler LFSR polynomial & $1+X^{14}+X^{15}$ \\
\hline Outer encoder & $\begin{array}{l}\mathrm{BCH}\left(K_{B C H}, N_{B C H}\right) \text { code using the codes listed } \\
\text { in Tables XV and XVI }\end{array}$ \\
\hline Inner encoder & $\begin{array}{l}\text { LDPC code with code rates } 1 / 2,3 / 5,2 / 3,3 / 4,4 / 5, \\
5 / 6\end{array}$ \\
\hline Bit interleaver & $\begin{array}{l}\text { parity interleaving followed by row-column inter- } \\
\text { leaving }\end{array}$ \\
\hline Modulation schemes & QPSK, 16-QAM, 64-QAM, 256-QAM \\
\hline Cell interleaver & pseudo-random permutation of data in a FEC frame \\
\hline Time interleaver & row-column interleaving \\
\hline
\end{tabular}

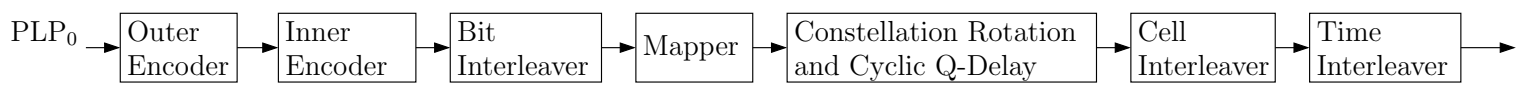
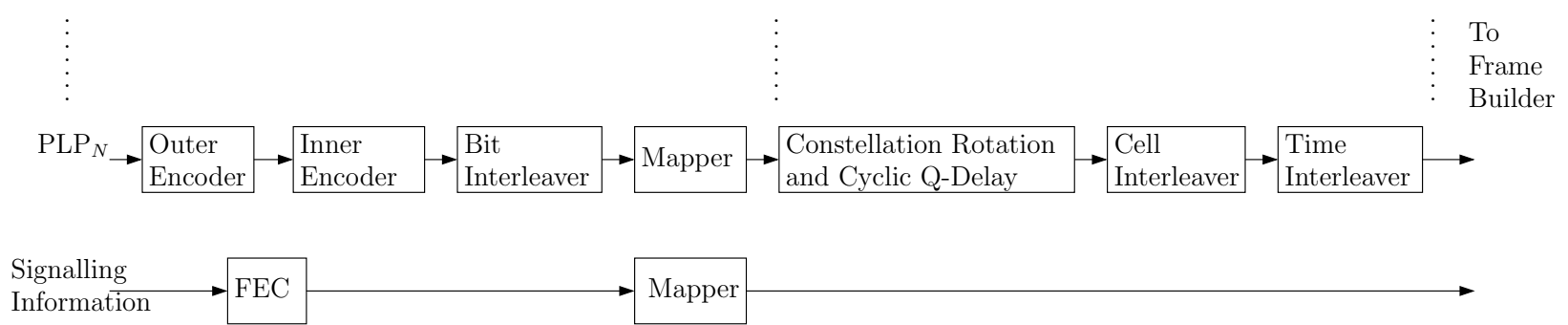

Fig. 17. Detailed schematic of the DVB-T2 BICM block of Figure 15 using the system parameters listed in Table XIV.

TABLE XV

DVB-T2 CODING PARAMETERS FOR 64800-BIT FRAMES [40].

\begin{tabular}{|l|c|c|c|c|}
\hline LDPC code rate & BCH input block $K_{B C H}$ & BCH coded block $N_{B C H}$ & BCH correction capability [bits] & LDPC coded Block \\
\hline $1 / 2$ & 32208 & 32400 & 12 & 64800 \\
\hline $3 / 5$ & 38688 & 38880 & 12 & 64800 \\
\hline $2 / 3$ & 43040 & 43200 & 10 & 64800 \\
\hline $3 / 4$ & 48408 & 48600 & 12 & 64800 \\
\hline $4 / 5$ & 51648 & 51840 & 12 & 64800 \\
\hline $5 / 6$ & 53840 & 54000 & 10 & 64800 \\
\hline
\end{tabular}

TABLE XVI

DVB-T2 CODING PARAMETERS FOR 16200-BIT FRAMES [40].

\begin{tabular}{|l|c|c|c|c|}
\hline LDPC code rate & BCH input block $K_{B C H}$ & BCH coded block $N_{B C H}$ & BCH correction capability [bits] & LDPC coded Block \\
\hline $1 / 4$ & 3072 & 3240 & 12 & 16200 \\
\hline $1 / 2$ & 7032 & 7200 & 12 & 16200 \\
\hline $3 / 5$ & 9552 & 9720 & 12 & 16200 \\
\hline $2 / 3$ & 10632 & 10800 & 12 & 16200 \\
\hline $3 / 4$ & 11712 & 11880 & 12 & 16200 \\
\hline $4 / 5$ & 12432 & 12600 & 12 & 16200 \\
\hline $5 / 6$ & 13152 & 13320 & 12 & 16200 \\
\hline
\end{tabular}


facilitate an improved system performance. It is also worth mentioning here that upon using rotated constellation, iterative decoding [37] can be carried out by exchanging extrinsic information between the demapper and the LDPC decoder in the receiver. This exchange of soft information allows us to attain an improved system performance. However, using the rotated constellations and iterative detection increases the receiver's implementation complexity.

Then, after the mapper, the symbols are interleaved by a cell interleaver followed by a time interleaver, as shown in Figure 17. The cell interleaver outputs a pseudo-random permutation of the cells in the FECFrame. On the other hand, the time interleaver of Figure 17 operates on what is referred to as Interleaving Frames (IF) and Time Interleaving Blocks (TIBlocks). Before describing the interleaving operations, it is important to understand the concept of FECFrames, IFs and TIBlocks. The FECFrame is, as mentioned before, the output of the mapper seen in Figure 17. On the other hand, the IF is an important concept in the DVB-T2 system. The IF is a framing structure which is performed for every PLP. An integer number of FECFrames is allocated per IF, which is made of one or more TIBlocks. On the other hand, a TIBlock has an integer number of FECFrames, but the number of FECFrames in a TIBlock is not constant in all IFs. The time interleaver is a row-column interleaver which operates on a TIBlock, where the data is written in columns and read in rows. It is also worth noting that the cell interleaver is a pseudo-random permutation which is initialised for the first FECFrame in a TIBlock.

The cell interleaver of Figure 17 uniformly spreads the channel errors along the entire FECFrame. This prevents the bursts of errors overwhelming the LDPC decoder and hence improves the attainable system performance in channels imposing packet erasures. On the other hand, the time interleaver of Figure 17 provides the bulk of interleaving and spreads the symbols in the same FECFrame across the entire TIBlock, which might spread the symbols of a FECFrame across several OFDM symbols. This provides protection against time- and frequency-selective fading channels.

As shown in Figure 17 the signalling information is also channel encoded. Albeit not shown in the figure, the signalling information is divided into two parts known as L1-pre as well as L1-post signalling and each of them is encoded separately. The L1-pre signalling includes side-information signalling needed for the correct demodulation of the L1-post signalling such as the modulation and coding mode used in the L1post signalling. Additionally, the L1-pre signalling includes vital side-information required for the decoding of the PLPs, such as the input stream type used, i.e. TS or GSE, and the specific pilot pattern used. Hence, the correct detection of L1-pre signalling is important for enabling the reception and decoding of the L1-post signalling information. On the other hand, the L1-post signalling includes the signalling information necessary for demodulating the common PLPs and data PLPs.

The L1-pre signalling is first BCH encoded and then LDPC encoded at a code rate of $1 / 4$ using the short frame length. Then, the L1-pre signalling bits are mapped to BPSK cells. On the other hand, the L1-post signalling is $\mathrm{BCH}$ encoded then LDPC encoded with code rate $1 / 2$ and using the short frame
TABLE XVII

DVB-T2 MAXIMUM ACHIEVABLE DATA RATE IN AN 8 MHZ CHANNEL USING THE 32K OFDM SYMBOL SIZE [40].

\begin{tabular}{|c|c|c|}
\hline Modulation & LDPC Code Rate & Max. Bit Rate [Mbit/sec] \\
\hline \multirow{6}{*}{ QPSK } & $1 / 2$ & 7.49 \\
\hline & $3 / 5$ & 9.00 \\
\hline & $2 / 3$ & 10.01 \\
\hline & $3 / 4$ & 11.27 \\
\hline & $4 / 5$ & 12.02 \\
\hline & $5 / 6$ & 12.53 \\
\hline \multirow{6}{*}{ 16-QAM } & $1 / 2$ & 15.03 \\
\hline & $3 / 5$ & 18.07 \\
\hline & $2 / 3$ & 20.10 \\
\hline & $3 / 4$ & 22.61 \\
\hline & $4 / 5$ & 24.13 \\
\hline & $5 / 6$ & 25.16 \\
\hline \multirow{6}{*}{ 64-QAM } & $1 / 2$ & 22.51 \\
\hline & $3 / 5$ & 27.06 \\
\hline & $2 / 3$ & 30.11 \\
\hline & $3 / 4$ & 33.87 \\
\hline & $4 / 5$ & 36.14 \\
\hline & $5 / 6$ & 37.68 \\
\hline \multirow{6}{*}{ 256-QAM } & $1 / 2$ & 30.08 \\
\hline & $3 / 5$ & 36.15 \\
\hline & $2 / 3$ & 40.23 \\
\hline & $3 / 4$ & 45.25 \\
\hline & $4 / 5$ & 48.29 \\
\hline & $5 / 6$ & 50.34 \\
\hline
\end{tabular}

length. The bits are then mapped to BPSK, QPSK, 16-QAM or 64-QAM cells. When 16-QAM or 64-QAM are used, the output of the LDPC is first bit interleaved, using the same bit interleaver used for the PLPs BICM, before mapping the bits to their corresponding cells.

According to Figure 15 and Figure 17, the output of the time interleaver is then passed to a frame builder and to the OFDM modulator for transmission. The frame builder combines the output of the time interleaver for each PLP with the signalling information into arrays of OFDM symbols. The frame building process is carried out according to the schedule generated by the scheduler in the Input Processing block of Figure 16. The frame builder then assembles the transmission frame using the signalling information and the data for all the common PLPs and data PLPs.

The output of the frame builder seen in Figure 15 is then interleaved by a frequency interleaver having a size equal to the OFDM symbol size, which can be $1024=1 \mathrm{~K}, 2 \mathrm{~K}$, $4 \mathrm{~K}, 8 \mathrm{~K}, 16 \mathrm{~K}$ and $32 \mathrm{~K}$ symbols in DVB-T2. The frequency interleaver is a pseudo-random block interleaver operating on OFDM symbols. The goal of the frequency interleaver is to scramble the data of the different PLPs, as opposed to the previous interleavers operating on the data of a single PLP. Additionally, the frequency interleaver assists in breaking up any undesirable artefacts between the regular bursts at the channel output and the structured nature of the time interleaver. After the frequency interleaving, pilots are inserted into the OFDM symbol for allowing synchronisation and tracking as well as channel estimation at the receiver. Then, the OFDM symbol is transformed to the time domain using the appropriate IFFT size selected for transmission.

Finally, to summarise, the DVB-T2 system is characterised by the following features:

1) The DVB-T2 system is an OFDM modulated system relying on the classic Cyclic Prefix (CP), which makes 
TABLE XVIII

DVB-T2 REQUIRED SNR FOR ACHIEVING A $10^{-7}$ BER AFTER LDPC DECODING IN A GAUSSIAN CHANNEL, WHILE USING THE NORMAL FRAME LENGTH OF 64800 BITS [40].

\begin{tabular}{|l|c|c|}
\hline Modulation & Code Rate & Required SNR [dB] \\
\hline \multirow{4}{*}{ QPSK } & $1 / 2$ & 1.0 \\
\cline { 2 - 3 } & $3 / 5$ & 2.3 \\
\cline { 2 - 3 } & $2 / 3$ & 3.1 \\
\cline { 2 - 3 } & $3 / 4$ & 4.1 \\
\cline { 2 - 3 } & $4 / 5$ & 4.7 \\
\cline { 2 - 3 } & $5 / 6$ & 5.2 \\
\hline \multirow{5}{*}{ 16-QAM } & $1 / 2$ & 6.0 \\
\cline { 2 - 3 } & $3 / 5$ & 7.6 \\
\cline { 2 - 3 } & $2 / 3$ & 8.9 \\
\cline { 2 - 3 } & $3 / 4$ & 10.0 \\
\cline { 2 - 3 } & $4 / 5$ & 10.8 \\
\hline \multirow{5}{*}{ 64-QAM } & $5 / 6$ & 11.4 \\
\cline { 2 - 3 } & $1 / 2$ & 9.9 \\
\cline { 2 - 3 } & $3 / 5$ & 12.0 \\
\cline { 2 - 3 } & $2 / 3$ & 13.5 \\
\cline { 2 - 3 } & $3 / 4$ & 15.1 \\
\cline { 2 - 3 } & $4 / 5$ & 16.1 \\
\hline \multirow{5}{*}{ 256-QAM } & $5 / 6$ & 16.8 \\
\cline { 2 - 3 } & $1 / 2$ & 13.2 \\
\cline { 2 - 3 } & $3 / 5$ & 16.1 \\
\cline { 2 - 3 } & $2 / 3$ & 17.8 \\
\cline { 2 - 3 } & $3 / 4$ & 20.0 \\
\cline { 2 - 3 } & $4 / 5$ & 21.3 \\
\hline & $5 / 6$ & 22.0 \\
\hline
\end{tabular}

it resilient to transmission over the dispersive terrestrial channel. The DVB-T2 standard defines a diverse range of OFDM symbol sizes, which -together with a range of possible CP durations- is capable of providing an improved bandwidth efficiency as well as a good performance in SFNs.

2) The DVB-T2 system uses a combination of $\mathrm{BCH}$ and LDPC codes as well as a range of bit-, cell-, timeand frequency-interleaving for providing an improved physical layer system performance. It also employs the concept of rotated constellations for significantly improving the system performance in frequency-selective channels.

3) The DVB-T2 system uses what is known as PLP for providing a mechanism to transmit several services' data, while employing different coding and modulation schemes per PLP in order to fine-tune the servicespecific robustness. In this case, the data encoding is configured for maintaining a constant output symbol rate, regardless of the specific FEC and modulation schemes used.

4) The DVB-T2 system provides a flexible framing structure which is signalled to the receiver using the L1-pre and L1-post signalling information.

5) The DVB-T2 standard also allows the optional use of the two-transmit antenna aided Multiple-Input SingleOutput (MISO) [37,59] concept for improving the reception in areas, where similar-strength signals are received from two different transmitters.

6) The DVB-T2 system is future-proof since it allows for time-frequency slicing, where several radio frequency channels can be combined for increasing the capacity and improving the attainable system performance. Additionally, the DVB-T2 framing structure defines Future
Extension Frames (FEF) as optional parts of the framing structure for allowing the definition of their content in the future.

7) The cell and time interleavers were designed in a way to increase the interleaver depth without requiring an increase in the memory of the de-interleaver in the receiver.

The DVB-T2 system is flexible, with hundreds of possible configurations resulting in different system performances and different data rates. Table XVII lists the maximum achievable FEC-coded data rate in an $8 \mathrm{MHz}$ channel for a 32K OFDM symbol size. As shown in the table, in this configuration the DVB-T2 system is capable of attaining a data rate in excess of $50 \mathrm{Mbit} / \mathrm{sec}$. Additionally, Table XVIII lists the best achievable performance in a Gaussian channel with the aid of perfect channel estimation and perfect synchronisation. The table lists the SNRs required for achieving a BER of $10^{-7}$ after the LDPC decoder, which is equivalent to attaining a BER of $10^{-11}$ after the $\mathrm{BCH}$ decoder. The SNR values listed in Table XVIII are used by the receiver designers as a benchmark for their receiver implementation.

As mentioned earlier, the DVB-T2 standard was designed as a flexible standard with a range of possible configurations. The aim is to allow the broadcaster to select their most suitable configuration according to the bit rate as well as the quality they aim to provide for their customers. For example, as shown in Table XVII, using a higher code rate results in a higher attainable bit rate, while this also requires a higher SNR for maintaining a given quality of service. Additionally, using a higher order modulation scheme also results in a higher achievable bit rate, while again requiring a higher SNR value for attaining the required quality of service.

Furthermore, DVB-T2 was designed for including a wide range of possible FFT sizes such as $1 \mathrm{~K}, 2 \mathrm{~K}, 4 \mathrm{~K}, 8 \mathrm{~K}, 16 \mathrm{~K}$ and $32 \mathrm{~K}$ subcarriers with a range of possible GIs. The selection of FFT size influences both the inter-carrier spacing and the symbol duration in time. Increasing the FFT size results in a narrower sub-carrier spacing and consequently in a longer symbol duration. On the other hand, a larger FFT size implies a longer symbol duration and hence a reduced GI overhead for a given GI duration. On the other hand, smaller FFT sizes can be used for achieving an improved performance in a fast fading channel. For example, for a fixed rooftop reception, $32 \mathrm{~K}$ and $16 \mathrm{~K}$ FFT sizes can be used as a larger FFT size, which would reduce the GI fraction and increase the available capacity. By contrast, for mobile reception, a lower FFT size such as $8 \mathrm{~K}$ or $4 \mathrm{~K}$ may be considered.

The diverse GIs, on the other hand, offer an extended range of spectral efficiencies, where using a lower GI results in a reduced GI overhead and hence more data can be transmitted in a given time slot. Furthermore, the extended range of FFT sizes and GIs offered by the DVB-T2 standard provides a flexibility for the SFN network design, where a trade off between the network capacity and the achievable performance should always be considered.

\section{CONCLUSIONS AND FUTURE TRENDS}

It has been more than a century since broadcasting was invented. Television really took off in the 1950s, when TV 
sets became affordable and the programs more entertaining. The broadcasting industry is expected to evolve further amidst the tremendous range of innovations in the area of communications technology and it is also expected to continue as being an essential player in bringing information to people.

In this paper we provided a survey of the different digital TV standards developed in different parts of the world. In Section III we described the transmitter design of the DVB standards developed for terrestrial, cable, satellite and handheld transmission. In Section IV we highlighted the transmitter structure of the ATSC and ATSC-M/H standards developed in the USA, followed by a description of the ISDB-T/ISBD-S standards developed in Japan and the ISDTV developed in Brazil, which are discussed in Section V. In Section VI we detailed the transmitter structure of the Chinese DTMB system. Finally, in Section VII we provided a detailed description of the DVB-T2 standards as a design example. Table XIX lists the different standards surveyed in this paper and the index of the figures showing their schematics as well as their parameter tables. Table XIX portrays the evolution of communications technology and how different technologies were adopted in the different DTV standards over time.

When designing communications systems, it is always beneficial to think in terms of the different design tradeoffs including their performance, implementation complexity, channel characteristics, coding rate, coding gain, system bandwidth and effective throughput. In what follows, we will compare and contrast the different architectures of the different DTV broadcasting standards in terms of these trade-offs. The main building blocks of the DTV broadcasting standards are the energy dispersal, the outer and inner FEC encoders, interleavers and modulators.

Energy dispersal is used for randomising the transmitted bit sequence for the sake of eliminating long sequences of ' 0 's and ' 1 's with the aid of LFSR. Different standards use different LFSR generator polynomials in order to achieve the same effect. The complexity of the randomisation process is dependent on the LFSR memory length and on the generator polynomial used; however, in practice the difference in complexity between implementing a more complicated LFSR generator polynomial or using a simpler one in trivial.

Most of the standards described in this paper use RS codes as the outer FEC code, while DVB-T2 uses BCH codes. Both $\mathrm{BCH}$ and RS codes are used for correcting the residual errors after the demodulation and/or inner FEC decoding. The RS codes are symbol based and hence they are capable of correcting bursts of error confined to a multi-bit symbol, while the $\mathrm{BCH}$ codes are binary codes designed for the correction of random errors. A disadvantage of the burst error correcting capability is that if the burst is spread over several symbols, then the RS code becomes incapable of correcting this burst, whereas the $\mathrm{BCH}$ code may still be able to correct the burst. The implementation complexity of $\mathrm{BCH}$ codes is lower than that of RS codes.

Interleavers disperse the bursts of errors which would potentially overwhelm the channel decoder. This improves the attainable system performance. However, using longer interleavers requires more memory for storing the data and imposes an increased delay on the system. In contrast to terrestrial and mobile channels, cable and satellite channels typically do not experience fading. Hence, a single interleaver is used in the cable and satellite standards, while several interleavers are invoked in the transmission chain of the terrestrial and hand-held standards. The interleavers in the terrestrial and hand-held standards help improve the system performance at the cost of imposing an increased delay on the system, as well as increasing the cost and complexity of the receiver implementation by requiring more processing power and more memory.

Due to multipath and fading channels, errors are introduced in the transmitted data and hence error correction codes are used for correcting these errors. Several types of inner FEC codes are used in the different standards described in this paper, including punctured convolutional codes and LDPC codes. The LDPC code's decoders are more complex than a convolutional code's Viterbi decoder. However, LDPC codes are capable of attaining an improved system performance compared to a convolutionally encoded system. Hence, it can be seen that the most recent DTV broadcasting standards use LDPC codes in order to attain a better performance and due to the fact that the advances in hardware technology made it feasible to implement an LDPC decoders at a reasonable implementation complexity and cost.

Finally, the modulator design is another important parameter when comparing the performance versus complexity of the communications systems. In cable and satellite channels, where the channel is typically non-dispersive, a single-carrier modulator is used. In terrestrial and hand-held channels, where the channel experiences severe fading in time and frequency, a more sophisticated modulator is required. Two options were used in the DTV broadcasting standards; OFDM was adopted in all standards except for ATSC, which uses VSB. The OFDM and VSB modulation techniques attain a similar performance, but interestingly, they have different implementation complexity. The OFDM demodulator is simple to implement and it is capable of supporting SFNs. On the other hand, VSB is a single-carrier modulation technique, which renders the equaliser design and implementation more complicated than that of OFDM. However, with the advances in equaliser technology, the VSB equaliser implementation becomes feasible while attaining a comparable performance to OFDM in multipath channels. On the other hand, 8-VSB is reminiscent of a single side-band transmission scheme, while OFDM is a double side-band technique, which means that 8 -VSB is potentially more bandwidth-efficient than OFDM. Additionally, it is worth noting that VSB was selected in ATSC to make the new ATSC standard compatible with the older NTSC scheme.

We live in the age of tablet PCs, smart-phones and Internet $\mathrm{TV}$, which have pushed the possibilities of entertainment to a whole new world and it is expected that the broadcasting industry will further evolve in order to match peoples' expectations and needs. Currently, the world is in the process of switching from analogue to digital broadcasting systems and the markets are slowly migrating toward HDTV. The digital TV market has been driven by the desire to achieve both a high picture quality and connectivity in the home.

In the past few years, consumer habits have been following 
TABLE XIX

SURVEY OF THE DTV STANDARDS IN THIS PAPER.

\begin{tabular}{|c|c|c|c|c|c|c|c|}
\hline Standard & Year & Video Codec & $\begin{array}{l}\text { Outer } \\
\text { FEC }\end{array}$ & Inner FEC & Interleavers & $\begin{array}{l}\text { Transmitter structure } \\
\text { and parameters }\end{array}$ & $\begin{array}{l}\text { Transmission } \\
\text { channel }\end{array}$ \\
\hline DVB-S & 1993 & MPEG-2 & RS code & $\begin{array}{l}\text { Punctured convo- } \\
\text { lutional codes }\end{array}$ & $\begin{array}{l}\text { Outer Convolutional } \\
\text { interleaver }\end{array}$ & Figure 1, Table II & Satellite \\
\hline DVB-C & 1994 & $\begin{array}{l}\text { MPEG-2 and } \\
\text { MPEG-4 }\end{array}$ & RS code & - & $\begin{array}{l}\text { Outer Convolutional } \\
\text { interleaver }\end{array}$ & Figure 1, Table II & Cable \\
\hline ATSC & 1996 & MPEG-2 & RS code & $\begin{array}{l}2 / 3 \text {-rate } 4 \text {-state } \\
\text { trellis encoder }\end{array}$ & $\begin{array}{l}\text { Outer symbol inter- } \\
\text { leaver, } \\
\text { Inner Convolutional } \\
\text { interleaver }\end{array}$ & Figure 6, Table VIII & Terrestrial \\
\hline ISDB-T & 1997 & MPEG-2 & RS code & $\begin{array}{l}\text { convolutional } \\
\text { code }\end{array}$ & $\begin{array}{l}\text { Outer Convolutional } \\
\text { interleaver, } \\
\text { Inner serial-to- } \\
\text { parallel conversion } \\
\text { followed by bit } \\
\text { delay }\end{array}$ & Figure 12 , Table XI & Terrestrial \\
\hline$\overline{\text { DVB-T }}$ & 2000 & $\begin{array}{l}\text { MPEG-2 and } \\
\text { H.264/MPEG-4 } \\
\text { AVC }\end{array}$ & RS code & $\begin{array}{l}\text { Punctured convo- } \\
\text { lutional codes }\end{array}$ & $\begin{array}{l}\text { Outer convolutional } \\
\text { interleaver, } \\
\text { Inner bit-wise } \\
\text { and symbol-wise } \\
\text { interleaver }\end{array}$ & $\begin{array}{l}\text { Figure 1, Table II, } \\
\text { Table III }\end{array}$ & Terrestrial \\
\hline ISDB-S & 2000 & MPEG-2 & RS code & $\begin{array}{l}\text { convolutional } \\
\text { code }\end{array}$ & $\begin{array}{l}\text { Outer row-column } \\
\text { interleaver }\end{array}$ & Figure 13 , Table XII & Satellite \\
\hline DVB-H & 2004 & $\begin{array}{l}\text { H.264/MPEG-4 } \\
\text { AVC }\end{array}$ & RS code & $\begin{array}{l}\text { Punctured convo- } \\
\text { lutional codes }\end{array}$ & $\begin{array}{l}\text { Outer convolutional } \\
\text { interleaver, } \\
\text { Inner bit-wise } \\
\text { and symbol-wise } \\
\text { interleaver }\end{array}$ & Figure 5, Figure 1 & Mobile \\
\hline DTMB & 2006 & $\begin{array}{l}\text { MPEG-2 and } \\
\text { H.264/MPEG-4 } \\
\text { AVC }\end{array}$ & RS code & LDPC code & $\begin{array}{l}\text { Outer row-column } \\
\text { byte interleaver, } \\
\text { Inner row-column } \\
\text { bit interleaver }\end{array}$ & Figure 14 , Table XIII & $\begin{array}{l}\text { Terrestrial } \\
\text { and Mobile }\end{array}$ \\
\hline ISDTV & 2006 & H.264/AVC & RS code & $\begin{array}{l}\text { convolutional } \\
\text { code }\end{array}$ & $\begin{array}{l}\text { Outer Convolutional } \\
\text { interleaver, } \\
\text { Inner serial-to- } \\
\text { parallel conversion } \\
\text { followed by bit } \\
\text { delay }\end{array}$ & Figure 12 , Table XI & $\begin{array}{l}\text { Terrestrial } \\
\text { and mobile }\end{array}$ \\
\hline J.83-B & 2007 & MPEG-2 & RS code & $\begin{array}{lr}\text { rate-7/8 } & 256- \\
\text { QAM } & \text { TCM } \\
\text { and rate-5/6 } \\
\text { 64-QAM TCM }\end{array}$ & $\begin{array}{l}\text { Outer Convolutional } \\
\text { Interleaver }\end{array}$ & Figure 11, Table X & Cable \\
\hline DVB-T2 & 2008 & $\begin{array}{l}\text { MPEG-2 and } \\
\text { H.264/MPEG-4 } \\
\text { AVC }\end{array}$ & $\mathrm{BCH}$ code & LDPC code & $\begin{array}{l}\text { bit interleaver, } \\
\text { cell interleaver, } \\
\text { time interleaver, } \\
\text { frequency interleaver }\end{array}$ & $\begin{array}{l}\text { Figure } 15 \text {, Figure } 16, \\
\text { Figure } 17 \text {, Table XIV }\end{array}$ & $\begin{array}{l}\text { Terrestrial } \\
\text { (potentially } \\
\text { Mobile) }\end{array}$ \\
\hline
\end{tabular}

new trends, including social networking and user-generated content, longer sessions relying on mobile devices such as smart phones and tablets. These trends were accompanied by the growing popularity of in-car entertainment, the demand for the ability to watch TV in the house as well as on the move and finally, the popularity of catch-up TV, video-on-demand and interactive applications.

A common theme of the above-mentioned trends is that of supporting mobility. Mobile TV is expected to make the digital TV a ubiquitous experience. Mobile TV is considered to be an evolving technology, which encompasses more than simply watching TV on a mobile device having a smaller screen. In addition to that, advanced features such as video on demand, time- and device-shifting, which facilitate viewing TV programs later in time and continue watching where you stopped even after switching to a different device, its intricate integration with social media is also anticipated. The mobile TV content can be delivered either by using regular broadcast television or by using the mobile network or in fact, by combining both.
When considering mobile reception, there are several technical challenges due to the hostile nature of the mobile channel, for supporting robust and reliable transmission. There are further forthcoming standards that are being developed around the globe including:

- Digital Video Broadcasting for Hand-held, also known as DVB-H, in Europe. At the time of writing, the standardisation process of a second generation hand-held standard is in an advanced stage.

- ATSC-M/H in North America.

- Terrestrial Digital Multimedia Broadcasting (T-DMB) in South Korea.

- Integrated Services Digital Broadcasting - Terrestrial Mobile Multimedia (ISDB-Tmm) in Japan.

- DTMB in China and Hong Kong.

- China Mobile Multimedia Broadcasting (CMMB) in China.

- 3GPP Multi-media Broadcast Multicast Service (MBMS).

Multiple-Input Multiple-Output (MIMO) techniques seem to 
be an inevitable technology that will be adopted in mobile TV. This is to provide a good system performance, while maintaining a high data rate in a mobile scenario and supporting high vehicular speeds. A combination of diversity and multiplexing oriented MIMO can be used in order to allow for providing a good performance as well as a high system throughput.

The future will be a convergence between the broadcast, mobile and broadband services. The term "convergence" is becoming popular, although it means different things to different people; it may simply mean the convergence of services, including broadcast services, mobile services and broadband services into a single network. We are now in an era, where the communication services can converge, with the migration of networks to IP-based technology. That is mainly due to the use of digital technology, the reduction in cost accompanied by the increase in computing power, the advances in data compression technology and the wide adoption of IP-based packet switching. All in all, it will be an exciting era for broadcast engineers and scientists.

\section{LIST OF ACRONYMS}

\begin{tabular}{|c|c|}
\hline ATSC & Advanced Television System Committee \\
\hline ATSC-M/H & $\begin{array}{l}\text { Advanced Television System Committee for } \\
\text { Mobile and Hand-held Transmission }\end{array}$ \\
\hline AWGN & Additive White Gaussian Noise \\
\hline $\mathrm{BB}$ & Base-Band \\
\hline $\mathrm{BCH}$ & Bose-Chaudhuri-Hocquenghem \\
\hline BICM & Bit-Interleaved Coded Modulation \\
\hline $\mathrm{CLCH}$ & Control Logical Channel \\
\hline CMMB & China Mobile Multimedia Broadcasting \\
\hline $\mathrm{CP}$ & Cyclic Prefix \\
\hline $\mathrm{CRC}$ & Cyclic Redundancy Check \\
\hline DTMB & $\begin{array}{l}\text { Digital Terrestrial Television Multimedia } \\
\text { Broadcasting }\end{array}$ \\
\hline DTV & Digital Television \\
\hline DVB & Digital Video Broadcasting \\
\hline DVB-T & $\begin{array}{l}\text { Digital Video Broadcasting for Terrestrial } \\
\text { Transmission }\end{array}$ \\
\hline DVB-T2 & $\begin{array}{l}\text { Second generation of Digital Video } \\
\text { Broadcasting for Terrestrial Transmission }\end{array}$ \\
\hline DVB-C & $\begin{array}{l}\text { Digital Video Broadcasting for Cable } \\
\text { Transmission }\end{array}$ \\
\hline DVB-C2 & $\begin{array}{l}\text { Second Generation of Digital Video } \\
\text { Broadcasting for Cable Transmission }\end{array}$ \\
\hline DVB-S & $\begin{array}{l}\text { Digital Video Broadcasting for Satellite } \\
\text { Transmission }\end{array}$ \\
\hline DVB-S2 & $\begin{array}{l}\text { Second Generation of Digital Video } \\
\text { Broadcasting for Satellite Transmission }\end{array}$ \\
\hline DVB-H & $\begin{array}{l}\text { Digital Video Broadcasting for Hand-held } \\
\text { Transmission }\end{array}$ \\
\hline ETSI & $\begin{array}{l}\text { European Telecommunications Standards } \\
\text { Institute }\end{array}$ \\
\hline FEC & Forward Error Correction \\
\hline FEF & Future Extension Frame \\
\hline FIC & Fast Information Channel \\
\hline GSE & Generic Stream Encapsulation \\
\hline HDTV & High Definition Television \\
\hline
\end{tabular}

IF

IP

IPTV

ISDB-T

ISDB-S

ITU

LDPC

LFSR

LOS

MBMS

MPE-FEC

NTSC

OFDM

PAL

PAPR

PAT

PCC

PCCC

PID

PLCH

PLP

PMT

PSD

PSI

QEF

RS

SDTV

SECAM

SFN

SLCH

SNR

TCM

T-DMB

TIBlock

TMCC

TPC

TS

TV
Interleaving Frame

Internet Protocol

Internet Protocol Television

Integrated Services Digital Broadcasting for Terrestrial Transmission

Integrated Services Digital Broadcasting for Satellite Transmission

International Telecommunications Union

Low Density Parity Check

Linear Feedback Shift Register

Line Of Sight

Multimedia Broadcast Multicast Service

Multi-Protocol data FEC

National Television System Committee

Orthogonal Frequency Division Multiplexing

Phase Alternating Line

Peak-to-Average Power Ratio

Program Association Table

Punctured Convolutional Code

Parallel Concatenated Convolutional Code

Packet ID

Physical Logical Channel

Physical Layer Pipe

Program Map Table

Power Spectral Density

Program-Specific Information

Quasi-Error Free

Reed-Solomon Code

Standard Definition Television

Sequential Couleur A Memiore

Single Frequency Network

Service Logical Channel

Signal-to-Noise Ratio

Trellis Coded Modulation

Terrestrial Digital Multimedia Broadcasting

Time Interleaving Block

Transmission and Multiplexing Configuration Control

Transmission Parameter Channel

Transport Stream

Television

\section{REFERENCES}

[1] "Broadcasting pioneers: The many innovators behind television history," http://inventors.about.com/library/inventors/bltelevision.htm.

[2] "Television history - the first 75 years," http://www.tvhistory.tv.

[3] W. Fischer, Digital Television: A practical Guide for Engineers. Springer-Verlag, 2004.

[4] M.S. Alencar, Digital TV Systems. Cambridge University Press, 2009.

[5] L. Hanzo, S.X. Ng, T. Keller and W. Webb, Quadrature Amplitude Modulation: From Basics to Adaptive Trellis-Coded, Turbo-Equalised and Space-Time Coded OFDM, CDMA and MC-CDMA Systems. Chichester, England: John Wiley and Sons Ltd and IEEE Press, NY, USA, second Edition, 2004.

[6] Y. Wu, S. Hirakawa, U.H. Reimers and J. Whitaker, "Overview of Digital Television Development Worldwide," Proc. IEEE, vol. 94, pp. 821, January 2006.

[7] "The world in 2011: ICT facts and figures," tech. rep., International Telecommunication Union (ITU), October 2011.

[8] J.H. Udelson, The Great Television Race: A History of the American Television Industry. University of Alabama Press, 1982. 
[9] M.S. Richer, G. Reitmeier, T. Gurley, G.A. Jones, J. Whitaker and R. Rast, "The ATSC Digital Television System," Proc. IEEE, vol. 94, pp. 37-43, January 2006.

[10] S. Haykin, "Cognitive radio: brain-empowered wireless communications," IEEE J. Sel. Areas Commun., vol. 23, pp. 201-220, February 2005.

[11] M. Fitch, M. Nekovee, S. Kawade, K. Briggs and R. Mackenzie, "Wireless service provision in TV white space with cognitive radio technology: A telecom operator's perspective and experience," IEEE Commun. Mag., vol. 49, pp. 64-73, March 2011.

[12] S.J. Shellhammer, A.K. Sadek and W. Zhang, "Technical challenges for cognitive radio in the TV white space spectrum," in Information Theory and Applications Workshop, pp. 323-333, February 2009.

[13] R.J. Crinon, D. Bhat, D. Catapano, G. Thomas, J.Y. Van Loo and G. Bang, "Data Broadcasting and Interactive Television," Proc. IEEE, vol. 94, pp. 102-118, January 2006.

[14] H. Asami and M. Sasaki, "Outline of ISDB Systems," Proc. IEEE, vol. 94, pp. 248-250, January 2006.

[15] L. Hanzo, P. Cherriman, J. Streit, Video Compression and Communications: From Basics to H.261, H.263, H.264, MPEG4 for DVB and HSDPA-Style Adaptive Turbo-Transceivers. John Wiley \& Sons - IEEE Press, 2007.

[16] G.A. Davidson, and M.A. Isnardi, L.D. Fielder, M.S. Goldman and C.C. Todd, "ATSC Video and Audio Coding," Proc. IEEE, vol. 94, pp. 60-76, January 2006.

[17] ISO/IEC International Standard IS 13818, "Generic coding of moving pictures and associated audio," November 2004.

[18] U. Ladebusch and C.A. Liss, "Terrestrial DVB (DVB-T): A Broadcast Technology for Stationary Portable and Mobile Use," Proc. IEEE, vol. 94, pp. 183-193, January 2006.

[19] European Telecommunications Standards Institute (ETSI), European Standard (Technical Specifications), "Digital Video Broadcasting (DVB) - Generic Stream Encapsulation (GSE) Protocol,” 2007.

[20] E. Tsekleves, J. Cosmas, A. Aggoun and J. Loo, "Converged Digital TV Services: The Role of Middleware and Future Directions of Interactive Television," International J. Digital Multimedia Broadcasting, vol. 2009, no. 643680, 2009.

[21] M. M. da Silva, Multimedia Communications and Networking. CRC Press, Taylor \& Francis Group, 2012.

[22] L. Hanzo and T. Keller, OFDM and MC-CDMA: A Primer. John Wiley \& Sons - IEEE Press, 2006.

[23] European Telecommunications Standards Institute (ETSI), European Standard (Telecommunications Series), "Digital Video Broadcasting (DVB): Framing Structure, Channel Coding and Modulation for 11/12 GHz Satellite Services," 1997.

[24] European Telecommunications Standards Institute (ETSI), European Standard (Telecommunications Series), "Digital Video Broadcasting (DVB): Framing Structure, Channel Coding and Modulation for Cable Services," 1998.

[25] European Telecommunications Standards Institute (ETSI), European Standard (Telecommunications Series), "Digital Video Broadcasting (DVB): Framing Structure, Channel Coding and Modulation for Digital Terrestrial Television," 2009.

[26] European Telecommunications Standards Institute (ETSI), "Digital Video Broadcasting (DVB) - DVB-H Implementation Guidelines," tech. rep., 2009.

[27] U.H. Reimers, "DVB-The Family of International Standards for Digital Video Broadcasting," Proc. IEEE, vol. 94, pp. 173-182, January 2006.

[28] J.G. Proakis, Digital communications. McGraw-Hill, 2001.

[29] L. Hanzo, T.H. Liew, B.L. Yeap, R.Y.S. Tee and S.X. Ng , Turbo Coding, Turbo Equalisation and Space-Time Coding: EXIT-Chart-Aided NearCapacity Designs for Wireless Channels, 2nd Edition. John Wiley \& Sons - IEEE Press, 2011.

[30] U. Reimers, DVB: The Family of International Standards for Digital Video Broadcasting, 2nd Edition. Springer, 2004.

[31] European Telecommunications Standards Institute (ETSI), European Standard (Telecommunications Series), "Digital Video Broadcasting (DVB): Transmission System for Handheld Terminals," 2009.

[32] G. Faria, J.A. Henriksson, E. Stare and P. Talmola, "DVB-H: Digital Broadcast Services to Handheld Devices," Proc. IEEE, vol. 94, pp. 194 209, January 2006.

[33] M. Kornfeld and G. May, "DVB-H and IP Datacast - Broadcast to Handheld Devices," IEEE Trans. Broadcast., vol. 53, pp. 161-170, March 2007.

[34] European Telecommunications Standards Institute (ETSI), European Standard (Telecommunications Series), "Digital Video Broadcasting (DVB) - Second generation framing structure, channel coding and mod- ulation systems for Broadcasting, Interactive Services, News Gathering and other broadband satellite applications (DVB-S2)," 2009.

[35] R. Stasiewicz , "DVB-S2 The New International Standard," tech. rep., CBC Technology Review, www.cbc.radiocanada.ca/technologyreview/pdf/issue3-dvb.pdf, 2007.

[36] A. Morello and V. Mignone, "DVB-S2 - ready for lift off," tech. rep., RAI, Radio-televisione Italiana, 2004.

[37] L. Hanzo, O. Alamri, M. El-Hajjar and N. Wu, Near-Capacity MultiFunctional MIMO Systems: Sphere-Packing, Iterative Detection and Cooperation. John Wiley \& Sons - IEEE Press, 2009.

[38] A. Morello and V. Mignone, "DVB-S2: The Second Generation Standard for Satellite Broad-Band Services," Proc. IEEE, vol. 94, pp. 210-227, January 2006.

[39] R. Steele, L. Hanzo, Mobile Radio Communications, 2nd Edition. John Wiley \& Sons - IEEE Press, 1999.

[40] European Telecommunications Standards Institute (ETSI), European Standard (Telecommunications Series), "Digital Video Broadcasting (DVB) - Frame structure channel coding and modulation for a second generation digital terrestrial television broadcasting system (DVB-T2)," 2011.

[41] European Telecommunications Standards Institute (ETSI), European Standard (Telecommunications Series), "Digital Video Broadcasting (DVB) - Frame structure channel coding and modulation for a second generation digital transmission system for cable systems (DVB-C2)," 2011.

[42] J. Robert, C. Schaaf and L. Stadelmeier, "DVB-C2 - The standard for next generation digital cable transmission," in IEEE International Symposium on Broadband Multimedia Systems and Broadcasting, pp. 15, May 2009.

[43] W. Fischer, Digital Video and Audio Broadcasting Technology: A Practical Engineering Guide, Thirs Edition. Springer, 2010.

[44] Advanced Television Systems Committee, "ATSC Digital Television Standard: Part 2 RF Transmission System Characteristics," January 2007.

[45] W. Bretl, W.R. Meintel, G. Sgrignoli, X. Wang, S.M. Weiss and K. Salehian, "ATSC RF, Modulation and Transmission," Proc. IEEE, vol. 94, pp. 44-59, January 2006.

[46] Advanced Television Systems Committee, "ATSC-Mobile DTV Standard, Part 2 - RF/Transmission System Characteristics," October 2009.

[47] ATSC Planning Team 2, "Final Report on ATSC 3.0 - Next Generation Broadcast Television," tech. rep., Advanced Television Systems Committee, 2011.

[48] P.J. Hearty, "Carriage of Digital Video and Other Services by Cable in North America," Proc. IEEE, vol. 94, pp. 148-157, January 2006.

[49] International Telecommunication Union - Telecommunication Standardisation Sector ITU-T, "Series J: Cable Networks and Transmission of Television, Sound Programme and Other Multimedia Signals - Digital transmission of television signals Digital multi-programme systems for television, sound and data services for cable distribution," 2007.

[50] Association of Radio Industries and Business (ARIB), Japan, "Terrestrial Intergrated Services Digital Broadcasting (ISDB-T) - Specification of channel coding, framing structure and modulation," September 1998.

[51] M. Takada and M. Saito, "Transmission System for ISDB-T," Proc. IEEE, vol. 94, pp. 251 -256, January 2006.

[52] ITU-R Recommendation BO.1408-1, "Transmission system for advanced multimedia services provided by integrated services digital broadcasting in a broadcasting-satellite channel," tech. rep., 2002.

[53] H. Katoh, "Transmission System for ISDB-S," Proc. IEEE, vol. 94 pp. 289-295, January 2006.

[54] China Standard for Radio Film and Television Industry, "Framing Structure, Channel Coding and Modulation for Digital Television Terrestrial Broadcasting System,” 2006.

[55] J. Song, Z. Yang, L. Yang, K. Gong, C. Pan, J. Wang and Y. Wu, "Technical Review on Chinese Digital Terrestrial Television Broadcasting Standard and Measurements on Some Working Modes," IEEE Trans. Broadcast., vol. 53, pp. 1-7, March 2007.

[56] Jian Song, Digital Television Terrestrial Multimedia Broadcasting (DTMB). IEEE Press, March 2010.

[57] L. Vangelista, N. Benvenuto, S. Tomasin, C. Nokes, J. Stott, A. Filippi, M. Vlot, V. Mignone and A. Morello, "Key technologies for next-generation terrestrial digital television standard DVB-T2," IEEE Commun. Mag., vol. 47, pp. 146 -153, october 2009.

[58] C. Abdel Nour and C. Douillard, "Rotated QAM Constellations to Improve BICM Performance for DVB-T2,” in IEEE 10th International Symposium on Spread Spectrum Techniques and Applications, pp. 354 359, August 2008. 
[59] S.M. Alamouti, "A simple transmit diversity technique for wireless communications," IEEE J. Sel. Areas Commun., vol. 16, no. 8, pp. 14511458, 1998.

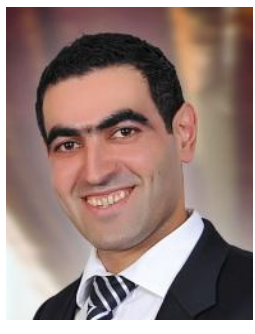

Mohammed El-Hajjar is a lecturer in the Electronics and Computer Science in the University of Southampton. He received his BEng degree in Electrical Engineering from the American University of Beirut, Lebanon in 2004. He then received an MSc in Radio Frequency Communication Systems and $\mathrm{PhD}$ in Wireless Communications both from the University of Southampton, UK in 2005 and 2008 , respectively. Following the $\mathrm{PhD}$, he joined Imagination Technologies as a research engineer, where he worked on designing and developing the BICM peripherals in Imagination's multi-standard communications platform, which resulted in several patent applications. In January 2012, he joined the Electronics and Computer Science in the University of Southampton as a lecturer in the Communications, Signal Processing and Control research group. He is the recipient of several academic awards and has published a Wiley-IEEE book and in excess of 40 journal and international conference papers. His research interests include mm-wave communications, massive MIMO, cooperative communications and Radio over fibre systems.

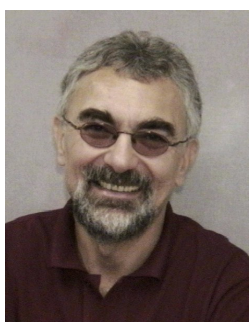

Lajos Hanzo (http://www-mobile.ecs.soton.ac.uk) FREng, FIEEE, FIET, Fellow of EURASIP, DSc received his degree in electronics in 1976 and his doctorate in 1983. In 2009 he was awarded the honorary doctorate "Doctor Honoris Causa" by the Technical University of Budapest. During his 35-year career in telecommunications he has held various research and academic posts in Hungary, Germany and the UK. Since 1986 he has been with the School of Electronics and Computer Science, University of Southampton, UK, where he holds the chair in telecommunications. He has successfully supervised $80 \mathrm{PhD}$ students, co-authored $20 \mathrm{John}$ Wiley/IEEE Press books on mobile radio communications totalling in excess of 10000 pages, published 1300 research entries at IEEE Xplore, acted both as TPC and General Chair of IEEE conferences, presented keynote lectures and has been awarded a number of distinctions. Currently he is directing a 100-strong academic research team, working on a range of research projects in the field of wireless multimedia communications sponsored by industry, the Engineering and Physical Sciences Research Council (EPSRC) UK, the European IST Programme and the Mobile Virtual Centre of Excellence (VCE), UK. He is an enthusiastic supporter of industrial and academic liaison and he offers a range of industrial courses. $\mathrm{He}$ is also a Governor of the IEEE VTS. During 2008 - 2012 he was the Editor-in-Chief of the IEEE Press and since 2009 he has been a Chaired Professor also at Tsinghua University, Beijing. For further information on research in progress and associated publications please refer to http://wwwmobile.ecs.soton.ac.uk 